\title{
Analytic description of semiclassical black-hole geometry
}

\author{
Pei-Ming Ho®, ${ }^{1, *}$ Yoshinori Matsuo, ${ }^{2, \dagger}$ and Yuki Yokokura ${ }^{3, \$}$ \\ ${ }^{1}$ Department of Physics and Center for Theoretical Physics, National Taiwan University, \\ Taipei 106, Taiwan, Republic of China \\ ${ }^{2}$ Department of Physics, Osaka University, Toyonaka, Osaka 560-0043, Japan \\ ${ }^{3}$ iTHEMS Program, RIKEN, Wako, Saitama 351-0198, Japan
}

(Received 23 April 2020; accepted 16 July 2020; published 31 July 2020)

\begin{abstract}
We study analytically the spacetime geometry of the black-hole formation and evaporation. As a simplest model of the collapse, we consider a spherical thin shell, and take the backreaction from the negative energy of the quantum vacuum state. For definiteness, we will focus on quantum effects of s-waves. We obtain an analytic solution of the semiclassical Einstein equation for this model, that provides an overall description of the black hole geometry form the formation to evaporation. As an application of this result, we find its interesting implication that, after the collapsing shell enters the apparent horizon, the proper distance between the shell and the horizon remains as small as the Planck length even when the difference in their areal radii is of the same order as the Schwarzschild radius. The position of the shell would be regarded as the same place to the apparent horizon in the semiclassical regime of gravity.
\end{abstract}

DOI: $10.1103 /$ PhysRevD.102.024090

\section{INTRODUCTION}

Quantum effects around the black holes have been well studied in the literature. A common feature of the quantum effects in many models is that there is an incoming negative vacuum energy flux around the apparent horizon [1,2], and the outgoing positive vacuum energy flux (i.e., Hawking radiation) appears well outside the apparent horizon. In this scenario, when the collapsing matter falls under the apparent horizon, there is no known mechanism to transfer all the information of the collapsing matter into Hawking radiation when the black-hole mass is still macroscopic, unless there are high-energy events around the apparent horizon $[3,4]$.

The goal of this paper is to have a better understanding of the spacetime geometry for black holes, in particular for the region inside the apparent horizon. We study the quantum effect of the vacuum energy-momentum tensor used in Refs. [1,2], and take its backreaction to the geometry into account. While there are works of numerical simulation, e.g., Ref. [5], we take the analytic approach to see detailed features of the geometry. As we will see, the analytic

\footnotetext{
*pmho@phys.ntu.edu.tw

matsuo@het.phys.sci.osaka-u.ac.jp

¥yuki.yokokura@ riken.jp
}

Published by the American Physical Society under the terms of the Creative Commons Attribution 4.0 International license. Further distribution of this work must maintain attribution to the author(s) and the published article's title, journal citation, and DOI. Funded by SCOAP. solution will allow us to learn detailed feature which is difficult to be found in numerical simulations. Throughout this paper, we focus on spherically symmetric configurations for simplicity.

An intuitive picture of the near-horizon geometry for a dynamical black hole is already known in the literature. With the backreaction of quantum effects taken into consideration, the black-hole geometry outside the collapsing matter has a wormholelike structure near the horizon due to the negative vacuum energy [6-11]. For black holes with spherical symmetry, it corresponds to a local minimum of the areal radius which we will refer to as the "neck" of the near-horizon geometry. The areal radius $a$ of the neck is approximately the Schwarzschild radius associated with the total mass of the collapsing matter. In the dynamical process of the evaporation, the neck is shrinking with time, and plays the role of the apparent horizon in the geometry. This picture has been clearly demonstrated in numerical simulation [5] as well as analytic calculation [12].

In this work, we aim at finding explicit analytic solutions to the semiclassical Einstein equation that are more explicit than existing results in the literature, with which one may explore new features of the black-hole geometry. As a simple example of the gravitational collapse, we consider a collapsing thin shell with the spherical symmetry. We shall provide explicit descriptions of the whole spacetime geometry, from the interior of the collapsing shell to large distances. We describe the geometry after the shell enters the apparent horizon, until the black hole is evaporated to a microscopic scale where the low-energy effective theory breaks down. 


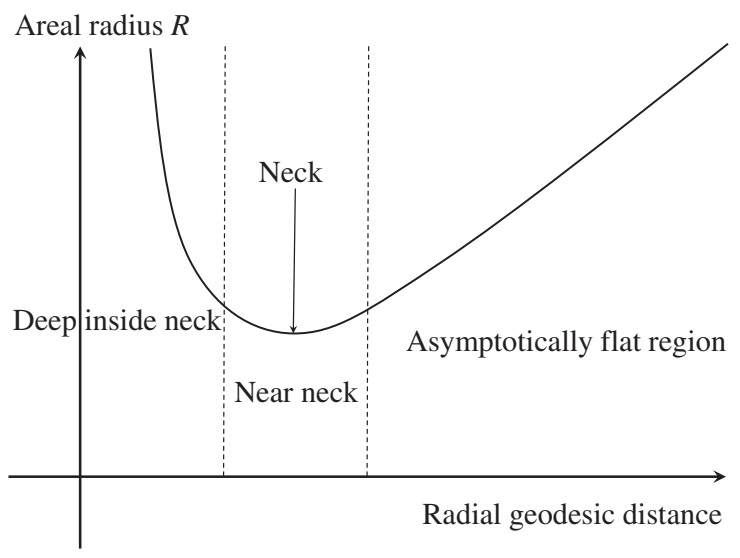

FIG. 1. The areal radius $R$ and 3 regions, asymptotically flat, near the neck and deep inside the neck. The areal radius has the local minimum, which is the apparent horizon in the dynamical case. The geometry near the neck is studied in [12], and the relation between its time evolution and the junction condition at the shell are discussed in Sec. III. The geometry outside the nearneck region is referred to as the asymptotically flat region. The geometry in this region will be analyzed by using the perturbative expansion around the outgoing Vaidya metric in Sec. IV. The areal radius becomes larger also inside the neck. We call this region as the region deep inside the neck and investigate the geometry in this region in Sec. V.

The geometry around the collapsing shell is obtained by connecting the interior and exterior geometries at the shell. The interior geometry is simply given by the flat space as it is not affected by the collapsing shell. The exterior geometry is the black hole geometry with backreaction, which is the semiclassical version of the (exterior) Schwarzschild metric and contains the near-horizon geometry explained above. We divide the exterior geometry into 3 regions: the asymptotically flat region, the region near the neck, and the region deep inside the neck (See Fig. 1). The semiclassical Einstein equation can be solved in different regions by different perturbative expansions. The initial condition is imposed in the past before the gravitational collapse, and the boundary conditions for each region are given by the junction conditions.

Our plan for this paper is as follows. In Sec. II, we review the static geometry for a black hole. The vacuum state, which is known as the Boulware vacuum, has no divergence because the black-hole geometry is modified from the classical solution by the backreaction from the negative vacuum energy. The exterior geometry of an evaporating black hole can then be obtained by introducing the Hawking radiation to the static black-hole geometry. In Sec. III, after reviewing the dynamical geometry outside the neck [12], we study the time evolution of the geometry and that of the shell. In Sec. IV, we consider the perturbative expansion in the asymptotically flat region and explore the junction condition to the region near the neck. The result suggests that the difference between the areal radius at the collapsing shell and that at the apparent horizon becomes large as the shell moves to the deeper region, but their proper distance remains as small as the Planck length. To further verify this result, we investigate the region deep inside the neck in Sec. V. The result is consistent with Sec. IV. Section VI is devoted to the conclusion and discussions.

\section{STATIC BLACK-HOLE GEOMETRY}

This section is a review of previous results included in Refs. $[8,9,12]$, where the s-wave approximation is used for the matter fields and the vacuum energy-momentum tensor is assumed to be given by the toy model proposed in Refs. [1,13] based on 2-dimensional massless scalar fields. ${ }^{1}$ (The solution was presented in different coordinate systems in Refs. [8,9], and here we use the same coordinate system as Ref. [12].) As a preliminary of the review, we introduce the vacuum energy-momentum tensor in Refs. [1,13] first.

We consider only spherically symmetric configurations in $4 \mathrm{D}$ spacetime. The metric can always be written as

$$
d s^{2}=-C(u, v) d u d v+R^{2}(u, v) d \Omega^{2},
$$

where $u$ and $v$ are retarded and advanced (null) time coordinates which span the 2-dimensional subspace of the temporal and radial directions, and $d \Omega^{2}$ is the metric on a unit 2-sphere,

$$
d \Omega^{2} \equiv d \theta^{2}+\sin ^{2} \theta d \phi^{2} .
$$

We refer to the radius $R(u, v)$ of the symmetric 2-spheres as the areal radius.

We assume that the semiclassical Einstein equation

$$
G_{\mu \nu}=\kappa\left\langle T_{\mu \nu}\right\rangle
$$

determines the spacetime geometry to a good approximation. The constant $\kappa$ is related to the Newton constant $G_{N}$ via $\kappa=8 \pi G_{N}$. Note that the quantum effect is taken into consideration through the vacuum expectation value $\left\langle T_{\mu \nu}\right\rangle$ of the energy-momentum tensor.

As mentioned above, we choose $\left\langle T_{\mu \nu}\right\rangle$ to be given by that of the 2D scalar fields $[1,13]$ as a concrete model. In vacuum, it is fixed by the conservation law and the Weyl anomaly up to integration constants $\beta(u)$ and $\gamma(v)$ as $^{2}[1,13]$

\footnotetext{
${ }^{1}$ The most general 4D static, spherically symmetric black-hole solutions with vacuum energy-momentum tensor of 4D conformal matters are studied in Ref. [11]. The toy model studied here is a special case belonging to the class of models with $q<0$ in Ref. [11].

${ }^{2}$ For simplicity, we have chosen the normalization factor $N$ to be $24 \pi$ so that $\alpha=\kappa$ in the notation of Ref. [12].
} 


$$
\begin{gathered}
\left\langle T_{u u}\right\rangle=-\frac{2}{R^{2}} C^{1 / 2} \partial_{u}^{2} C^{-1 / 2}+\frac{\beta(u)}{R^{2}}, \\
\left\langle T_{v v}\right\rangle=-\frac{2}{R^{2}} C^{1 / 2} \partial_{v}^{2} C^{-1 / 2}+\frac{\gamma(v)}{R^{2}}, \\
\left\langle T_{u v}\right\rangle=-\frac{1}{R^{2} C^{2}}\left[C \partial_{u} \partial_{v} C-\partial_{u} C \partial_{v} C\right], \\
\left\langle T_{\theta \theta}\right\rangle=0,
\end{gathered}
$$

where $C$ and $R$ are functions defined in the metric (2.1).

At large distances where

$$
r-a \gg \frac{\kappa}{a},
$$

$\left\langle T_{\mu \nu}\right\rangle$ should be very small, and the metric is approximated by the Schwarzschild metric,

$$
d s^{2}=-\left(1-\frac{a}{r}\right) d t^{2}+\frac{d r^{2}}{1-\frac{a}{r}}+r^{2} d \Omega^{2},
$$

where $a$ is the Schwarzschild radius. However, the perturbative correction is in general not weak around the Schwarzschild radius when [11]

$$
r-a \sim \mathcal{O}\left(\frac{\kappa}{a}\right) .
$$

\section{A. Geometry around the neck}

The vacuum state for the static geometry, which is known as the Boulware vacuum, has neither incoming nor outgoing radiation at large distances. It is given by the condition

$$
\beta(u)=0, \quad \gamma(v)=0
$$

in the vacuum energy-momentum tensor (2.4)-(2.7). It is well known that the vacuum energy-momentum tensor of the Boulware vacuum diverges at the horizon in the perturbative calculation around the Schwarzschild background. But, in fact, no divergence appears if the backreaction of the vacuum energy is properly taken into account [8]. Furthermore, the effect of the Hawking radiation in the Unruh vacuum $(\beta(u) \neq 0)$ only introduces slow $u$-dependence to this nonsingular static geometry in the parameters, such as the Schwarzschild radius [12]. As the dynamical geometry on each time slice has the same structure to the static geometry, we review the static geometry near the Schwarzschild radius $[8,9,12]$.

Here, we consider the geometry near the Schwarzschild radius with the backreaction from the vacuum energymomentum tensor. For static metrics, it is convenient to use the time coordinate associated with the timelike Killing vector. Since the areal radius $R$ may not be a single-valued function in the radial direction, it is better to take a different radial coordinate. In terms of the tortoise coordinate $x$ for the radial direction, the metric Eq. (2.1) is expressed as

$$
d s^{2}=-C(x)\left(d t^{2}-d x^{2}\right)+R^{2}(x) d \Omega^{2} .
$$

At large distances, we can consider the $\kappa$-expansion around the Schwarzschild solution

$$
C \simeq 1-\frac{a}{R}
$$

In the near-horizon region where the areal radius $R$ is very close to the Schwarzschild radius, $R-a \sim \mathcal{O}(\kappa / a)$, the Schwarzschild solution becomes comparable to its quantum correction.

We should define the $\kappa$-expansion in the near-horizon region $\mathrm{as}^{3}$

$$
\begin{gathered}
C(x)=\kappa C_{0}(x)+\mathcal{O}\left(\kappa^{2} / a^{4}\right), \\
R(x)=a+\kappa R_{0}(x)+\mathcal{O}\left(\kappa^{2} / a^{3}\right) .
\end{gathered}
$$

In the following, from time to time, $\mathcal{O}\left(\kappa^{n} / a^{m}\right)$ will be denoted as $\mathcal{O}\left(\kappa^{n}\right)$ for simplicity, as the power $m$ of $a$ is uniquely determined by the dimension of the quantity.

The solution to the semiclassical Einstein equations to the 1 st order has $[8,9,12]$

$$
\begin{gathered}
C_{0}=c_{0} e^{k x}, \\
R_{0}=a_{1}+\frac{c_{0}}{a k^{2}} e^{k x}-\frac{k}{4 a} x
\end{gathered}
$$

To determine the parameters $k, c_{0}$, and $a_{1}$, we consider the continuation of this solution to the region where the $\kappa$-expansion around the Schwarzschild solution is valid. ${ }^{4}$

It was pointed out in Ref. [11] that, in terms of the coordinate $z$ defined by

$$
d s^{2}=-C d t^{2}+d z^{2}+R^{2} d \Omega^{2},
$$

the perturbative expansion around the Schwarzschild solution can be extended to the near-horizon region where $R-a \sim \mathcal{O}(\kappa / a)$. The expansion to the first-order correction gives [11]

\footnotetext{
${ }^{3}$ The same ansatz is applicable for the near-horizon geometry in other static vacua $[8,11]$. For example, the solution for the Hartle-Hawking vacuum is given by a similar form as (2.16)(2.17) without the third term of (2.17).

${ }^{4}$ In Ref. [12], these parameters are not determined from the junction condition. Instead, they are fixed by assuming the relation $\dot{a}(u)=-\kappa \beta(u)$ between the time evolution of the Schwarzschild radius $a(u)$ and the Hawking radiation $\beta(u)$.
} 


$$
\begin{gathered}
C=\frac{z^{2}}{4 a^{2}}+\cdots, \\
R^{2}=a^{2}+\frac{1}{2} z^{2}+\kappa a_{2}-\kappa \log \frac{z}{a}+\cdots,
\end{gathered}
$$

near the Schwarzschild radius. Although the first-order correction to the areal radius $R$ is comparable to the 0thorder term (given by the Schwarzschild solution) at small $z$, no higher-order correction is of a comparable order of magnitude [11]. Hence, after the coordinate transformation to the tortoise coordinate, the solution above reproduces Eqs. (2.16) and (2.17).

In this way, the parameters in Eqs. (2.16) and (2.17) are fixed such that

$$
\begin{gathered}
C=\frac{1}{4} e^{\left(x-x_{0}\right) / a}+\cdots, \\
R^{2}=a^{2}+\frac{a^{2}}{2} e^{\left(x-x_{0}\right) / a}-\frac{\kappa}{2 a}\left(x-x_{0}\right)+\cdots,
\end{gathered}
$$

where the constant part of the 1 st-order terms in $R^{2}$ is absorbed by a redefinition of $a$. (Without loss of generality, one can set $x_{0}$ to 0 .)

The result above implies that the areal radius $R$ has a local minimum at

$$
x_{\text {neck }}=x_{0}-a \log \left(\frac{a^{2}}{\kappa}\right) \text {, }
$$

where

$$
R^{2}\left(x_{\text {neck }}\right)=a^{2}+\frac{\kappa}{2}\left(\log \frac{a^{2}}{\kappa}+1\right)+\mathcal{O}\left(\kappa^{2}\right) .
$$

In the following, we will refer to this local minimum of $R$ as the "neck."

Around the neck, there is no event horizon and the vacuum energy-momentum tensor has no divergence. However, Eqs. (2.21) and (2.22) provide a good approximation of the metric only in the near-horizon region where $R-a \sim \mathcal{O}(\kappa)$. In the next subsection, we consider the geometry deeper inside the neck.

\section{B. Geometry deep inside the neck}

In the previous subsection, we reviewed the static spacetime geometry in vacuum around the neck of a black hole. Here we turn to the region deep inside the neck (but still in vacuum) [9], assuming that the surface of the star is further deeper inside the neck.

Since the areal radius $R$ is a local minimum at the neck, it becomes larger as we go deeper inside the neck. When $R$ is close to the Schwarzschild radius so that $R-a \sim \mathcal{O}(\kappa)$, the solution (2.21)-(2.22) is a good approximation. In this subsection, we consider the geometry deeper inside the neck where

$$
R-a \sim \mathcal{O}\left(\kappa^{0}\right) .
$$

Although we refer to this region as "deep inside the neck," it does not imply that the proper distance in the radial direction between the neck and a point in this region is much larger than the Planck length. In fact, we will see in Sec. VD that it is of the order of the Planck length.

If we naively extend the solution (2.21)-(2.22) for $R-a \sim \mathcal{O}(\kappa)$ to the deeper region where $R-a \sim \mathcal{O}\left(\kappa^{0}\right)$, we deduce from Eq. (2.22) that

$$
\left|x-x_{0}\right| \sim \mathcal{O}\left(\kappa^{-1}\right)
$$

(note that $x-x_{0}<0$ inside the neck), so that $\log C \sim$ $\mathcal{O}\left(\kappa^{-1}\right)$. Thus, we expect that $C$ behaves as $\log C \sim \mathcal{O}\left(\kappa^{-1}\right)$ for $R-a \sim \mathcal{O}\left(\kappa^{0}\right)$. Defining $\rho$ by

$$
C=e^{2 \rho},
$$

we have the expansions

$$
\begin{aligned}
& \rho=\kappa^{-1} \rho_{0}+\rho_{1}+\mathcal{O}(\kappa), \\
& R=R_{0}+\kappa R_{1}+\mathcal{O}\left(\kappa^{2}\right),
\end{aligned}
$$

deep inside the neck.

The solutions to the leading-order terms of the semiclassical Einstein equation are found to be

$$
\begin{gathered}
\rho_{0}=-c_{1}\left(x-x_{0}\right)+c_{2}, \\
R_{0}^{2}=c_{1}\left(x-x_{0}\right)+c_{3},
\end{gathered}
$$

where $c_{1}, c_{2}$, and $c_{3}$ are integration constants.

The result above implies that (2.21)-(2.22) give a good approximation at the leading order even in this region. The patching of this solution with Eqs. (2.21)-(2.22) around the neck demands that

$$
c_{1} \simeq-\frac{\kappa}{2 a}, \quad c_{2} \simeq 0, \quad c_{3} \simeq a^{2} .
$$

It may appear strange that $c_{1}$ is of the 1 st order in $\kappa$, while, by definition, $R_{0}$ should be of the 0 th order. This is simply a result of Eq. (2.26) in this region. In terms of a properly rescaled coordinate such as $\tilde{x}=\kappa\left(x-x_{0}\right)$, all the parameters and coordinates are of $\mathcal{O}\left(\kappa^{0}\right) .^{5}$

In the next section, we will study the geometry for the Unruh vacuum by using the derivative expansion for the

\footnotetext{
${ }^{5}$ One may say that $x$ is not a good coordinate to describe the solution in this region, and $\tilde{x}$ is more appropriate, but the results are equivalent.
} 
time derivative $\partial_{u} \sim \mathcal{O}(\kappa)$. The expansion would not be valid for the geometry deep inside the neck which is studied in this subsection, since (2.26) implies that the $x$-derivative will also be very small, $\partial_{x} \sim \mathcal{O}(\kappa)$, effectively. Therefore, we cannot use the same (derivative) expansion for this region. We will consider another analysis for the geometry deep inside the neck, without using the derivative expansion, in Sec. V.

\section{COLLAPSING SHELL}

The geometry around the neck for a static black hole in the Boulware vacuum was discussed above in Sec. II A. In this section, we study the dynamical geometry around the neck for a black hole with the backreaction from the vacuum energy-momentum tensor for the Unruh vacuum. The geometry is obtained by solving the semiclassical Einstein equation in the $\kappa$-expansion for $\beta(u) \neq 0$. It turns out that the solution at the leading order takes the same form as the static case of Sec. II A, but the effect of the Hawking radiation introduces slow time evolution of the parameters, e.g., the Schwarzschild radius. The slow time evolution can be interpreted as a perturbation to the static geometry. Our analysis will fail around the end of the evaporation since the Hawking radiation will be very strong and time evolution will no longer be slow. A fully quantum treatment of gravity would be necessary around the end of the evaporation and it is out of the scope of this work. We will focus on the period earlier than the last moment of the evaporation $u \ll u_{c}$, where $u_{c}$ is the time of the complete evaporation, by using coordinates analogous to the outgoing Eddington-Finkelstein coordinates. ${ }^{6}$

As a simple model for the collapsing matter, we consider a thin shell falling at the speed of light. The matter distribution in the radial direction is approximated by the Dirac delta function. ${ }^{7}$ We will study the dynamical geometry outside the collapsing matter [12]. The geometry for a collapsing thin shell can be obtained by connecting this exterior geometry with the interior geometry (which is flat spacetime) at the collapsing shell. We will first consider the generic amplitude $\beta(u)$ of Hawking radiation, and then determine the Hawking radiation by imposing the junction condition on the collapsing shell.

\footnotetext{
${ }^{6} \mathrm{~A}$ similar structure can be found for the vacuum energymomentum tensor on the Schwarzschild geometry without the backreaction. The Boulware vacuum diverges at the future horizon while the Unruh vacuum is smooth there. The difference of two vacua becomes very large and cannot be treated as a perturbation near the future horizon. By using the outgoing Eddington-Finkelstein coordinates, which does not cover the future horizon, we can naturally focus on the other region where the Unruh vacuum can be interpreted as a perturbation of the Boulware vacuum.

${ }^{7}$ An infinitesimally thin shell introduces unphysical solutions [14] but they are avoided in our perturbative approach.
}

\section{A. Geometry outside collapsing shell}

The geometry outside the shell is a solution to the semiclassical Einstein equation (2.5)-(2.7). We impose the initial condition that there are no incoming matter excitations in the past null infinity other than the collapsing null shell. By identifying the null coordinates $u$ and $v$ with those in the asymptotically flat spacetime with $C \rightarrow 1$, the integration constant $\gamma(v)$ in the incoming energy flow $\left\langle T_{v v}\right\rangle$ (2.5) should vanish:

$$
\gamma(v)=0,
$$

as we assumed (2.11) for the static solution. The other integration constant $\beta(u)$ in the outgoing energy flow $\left\langle T_{u u}\right\rangle$ (2.4) is identified with the Hawking radiation and should be determined by the junction condition across the collapsing matter.

As the $u$-dependence of the dynamical solution is turned on by the nonzero $\beta(u)$, which contributes to the semiclassical Einstein equation at $\mathcal{O}(\kappa)$, the $u$-dependence of the solution must be very weak so that we can consider the derivative expansion in powers of $\partial_{u}$, with $\partial_{u} \sim \mathcal{O}(\kappa){ }^{8}$ This assumption should be physically reasonable since the evaporation of the black hole due to the Hawking radiation is very slow. The geometry is approximately static and the $u$-dependence can appear only in the higher-order corrections from the viewpoint of an observer in the asymptotically flat region.

Now, the semiclassical Einstein equation can be solved by using $\kappa$-expansion. We first make an ansatz for the nearhorizon region in a similar fashion to the static case

$$
\begin{gathered}
C(u, v)=\kappa C_{0}(u, x)+\mathcal{O}\left(\kappa^{2} / a^{4}\right), \\
R(u, v)=a(u)+\kappa R_{0}(u, x)+\mathcal{O}\left(\kappa^{2} / a^{3}\right),
\end{gathered}
$$

where $x$ is viewed as a function of $u$ and $v$ :

$$
x=\frac{1}{2}(v-u)+x_{0}(u) .
$$

The variables $a(u), C_{0}(u, x)$, and $R_{0}(u, x)$ all depend on $u$. They are functions of $\mathcal{O}\left(\kappa^{0}\right)$, while their $u$-derivatives, $\dot{a}(u), \partial_{u} C_{0}(u, x)$, and $\partial_{u} R_{0}(u, x)$, are assumed to be of $\mathcal{O}(\kappa)$. It is straightforward to solve the semiclassical Einstein equation for the Unruh vacuum, $\beta(u) \neq 0$ and $\gamma=0$, order by order in the $\kappa$-expansion. At the leading

\footnotetext{
${ }^{8}$ More precisely, in the $(t, x)$ coordinate system, the static solution (2.12) depends on $x$ but not on $t$. Clearly, the same solution can be written in the $(u, x)$ coordinate system with no dependence on $u$. When $\beta(u)$ is turned on at $\mathcal{O}\left(\kappa^{0}\right)$, the semiclassical Einstein equation implies a $u$-dependence with $\partial_{u} \sim \mathcal{O}(\kappa)$. This is not true in the $(u, v)$ coordinate system. Whenever we say $\partial_{u} \sim \mathcal{O}(\kappa)$, the $u$-derivative is taken with a fixed radial coordinate.
} 
order, $(v, v)$ and $(u, v)$-components of the semiclassical Einstein equation is exactly the same as those for the Boulware vacuum, as $u$-derivatives contribute only to the next-to-leading order. The Hawking radiation contributes only to the other part of the Einstein equation which gives a constraint on the integration constants. Therefore, the solution takes the same form as that in the static case, (2.21) and (2.22), but the integration constants are now $u$-dependent functions, ${ }^{9}$

$$
\begin{aligned}
C(u, v)=\frac{1}{4} e^{\left(x-x_{0}(u)\right) / a(u)}+\mathcal{O}\left(\kappa^{2}\right), \\
R(u, v)=a(u)+\kappa a_{1}(u)+\frac{a(u)}{4} e^{\left(x-x_{0}(u)\right) / a(u)} \\
-\frac{\kappa}{4 a(u)^{2}}\left(x-x_{0}(u)\right)+\mathcal{O}\left(\kappa^{2}\right) .
\end{aligned}
$$

where $x$ is now viewed as a function of $u$ and $v$ :

$$
x=\frac{1}{2}(v-u)+x_{0}(u) .
$$

For more details on the derivation and higher-order corrections, see [12]. The integration constant $a_{1}(u)$ can be absorbed by a redefinition of the Schwarzschild radius $a(u)$ and hence we can set it to 0 . The reference point $x_{0}(u)$ of the $x$-coordinate can be absorbed by a coordinate transformation of $u$. But we shall choose $u$ to agree with the $u$-coordinate in the asymptotically flat region, so $x_{0}(u)$ will not be set to zero. Note that the assumption $\partial_{u} \sim \mathcal{O}(\kappa)$ now implies that $\dot{a}(u)=\mathcal{O}(\kappa)$ and $\dot{x}_{0}(u)=\mathcal{O}(\kappa)$, where the dots on $\dot{a}$ and $\dot{x}_{0}$ stand for the $u$-derivative. The junction condition is consistent with these conditions as we will see below.

The semiclassical Einstein equation gives constraints on the boundary condition on a Cauchy surface. They are the differential equations for the integration constants, $a(u)$ and $x_{0}(u)$. At the leading order, we have

$$
\dot{a}(u)=-\kappa \beta(u)+\mathcal{O}\left(\kappa^{2}\right) .
$$

Thus, the condition $\dot{a}(u)=\mathcal{O}(\kappa)$ is consistent with the junction condition if $\beta(u)=\mathcal{O}\left(\kappa^{0}\right)$. The other integration constant $x_{0}(u)$ should be fixed by the coordinate patch to the asymptotically flat region for the $u$-coordinate.

The areal radius has a local minimum on constant- $u$ slices at

$$
x_{A}(u)=x_{0}(u)-a(u) \log \left(\frac{a^{2}(u)}{\kappa}\right),
$$

which coincides with Eq. (2.23), except that the parameters depend on $u$ here. Since the areal radius is always decreasing

\footnotetext{
${ }^{9}$ Although the integration constants depend on $u$, their $u$ derivatives are $\mathcal{O}(\kappa)$.
}

with $u$, along constant $-v$ lines, $x=x_{A}(u)$ is the trapping horizon (the trajectory of the apparent horizon).

The solution (3.5)-(3.6) must satisfy the ansatz (3.2)(3.3) above, or equivalently,

$$
C(u, v)=\mathcal{O}(\kappa), \quad R(u, v)=a(u)+\mathcal{O}(\kappa) .
$$

Therefore, (3.5)-(3.6) are not valid for arbitrary $u$ and $v$, but only in some specific regions. By using the position of the apparent horizon $x_{A}(u)$, the validity range of the perturbative expansion (3.2)-(3.3), or equivalently, (3.10), is the region

$$
x-x_{A}(u)=\mathcal{O}(a) .
$$

It should be noted that the derivative expansion is valid at each moment of $u$, and hence Eqs. (3.5) and (3.6) are valid for arbitrary $u$ as long as Eq. (3.11) is satisfied.

Since the solution (3.5)-(3.6) takes the same form as that for the static case for the Boulware vacuum, but the Hawking radiation introduces only the slow $u$-dependence, the Hawking radiation can be considered as a perturbation to the Boulware vacuum. ${ }^{10}$ It is also possible to treat $u$ dependent parts as perturbations, by using the expansion $a(u)=a\left(u_{1}\right)+(\Delta u) \dot{a}\left(u_{1}\right)+\cdots$ where $u_{1}$ is a moment of the retarded time $u$. Thus the solution (3.5)-(3.6) itself can be interpreted as a perturbation of the static solution as long as the difference of the retarded time $\Delta u$ from the moment $u_{1}$ is not too long and satisfies $\Delta u=\mathcal{O}(a)$. Although the solution cannot be approximated by the expansion by using a constant Schwarzschild radius (or equivalently that at $u=u_{1}$ ) if the duration $\Delta u$ is longer, the expression (3.5)(3.6) with $u$-dependent Schwarzschild radius is valid for arbitrary moments of time $u$ except for the end of the evaporation. The perturbation in fact breaks down around the end of the evaporation, when the Hawking radiation $\beta(u)$ will be much larger than $\mathcal{O}\left(\kappa^{0}\right)$. A fully quantum gravity theory would also be necessary then, and hence it is out of the scope of this work.

The solution (3.5)-(3.6) is valid only near the apparent horizon (3.11). In the deeper region, $R-a=\mathcal{O}(a)$, the geometry for the Unruh vacuum cannot be obtained by the perturbation from the Boulware vacuum. We will see the geometry in the deeper region in Sec. V.

\footnotetext{
${ }^{10}$ To be more precise, the solution (3.5)-(3.6) is not obtained as a perturbation around the static solution for the Boulware vacuum. However, we can in fact introduce the Hawking radiation as a perturbation by using the double expansion in $\kappa$ and $\epsilon$ with $\epsilon \ll 1$, where $\epsilon$ is introduced as $\beta(u)=\epsilon \beta_{0}(u)$. The solution at the 0 th order of the $\epsilon$-expansion is nothing but the static solution and hence it is a perturbation around the static solution. The solution (3.5)-(3.6) agrees with that of the double expansion with $\epsilon \rightarrow 1$, at the leading order. In this sense, the effect of the Hawking radiation in the solution (3.5)-(3.6) can be viewed as the perturbation to the Boulware vacuum.
} 


\section{B. Energy conservation on collapsing shell}

For the dynamical geometry outside the collapsing shell given by Eqs. (3.5)-(3.6), $\left\langle T_{u v}\right\rangle(2.6)$ is calculated as ${ }^{11}$

$$
\left\langle T_{u v}\right\rangle=-\frac{C(u, v)}{2 a^{2}(u) R^{2}(u, v)}+\mathcal{O}\left(\kappa^{2}\right) .
$$

while

$$
\left\langle T_{u v}\right\rangle=0
$$

in the flat spacetime inside the collapsing shell.

Without loss of generality, we will choose the collapsing shell to be located at $v=0$, and it passes through the point $x=x_{0}$ at $u=0$. The energy-momentum tensor $\left\langle T_{u v}\right\rangle$ is expressed in terms of the step function $\Theta$ as

$$
\left\langle T_{u v}\right\rangle=-\frac{C}{2 a^{2}(u) R^{2}(u, v)} \Theta(v)+\mathcal{O}\left(\kappa^{2}\right) .
$$

The conservation law

$$
\partial_{u}\left(R^{2}\left\langle T_{v v}\right\rangle\right)=-C \partial_{v}\left(C^{-1} R^{2}\left\langle T_{u v}\right\rangle\right),
$$

gives

$$
\partial_{u}\left\langle T_{v v}\right\rangle=\frac{C}{2 a(u)^{2} R^{2}(u, v)} \delta(v)+\cdots,
$$

where we only keep the term proportional to the delta function. Equation (3.16) implies that the energy on the collapsing shell $\left\langle T_{v v}\right\rangle$ is increasing, as the right-hand side of Eq. (3.16) is positive, despite the fact that the total mass of the black hole is decreasing. The increase of the energy on the shell comes from the vacuum energy sitting on top of the shell, and the total mass of the black hole decreases due to the negative vacuum energy inflow.

The other component of the conservation equation is

$$
\partial_{v}\left(R^{2}\left\langle T_{u u}\right\rangle\right)=-C \partial_{u}\left(C^{-1} R^{2}\left\langle T_{u v}\right\rangle\right) .
$$

If the collapsing shell directly loses energy into the Hawking radiation, $\left\langle T_{u и}\right\rangle$ must have a discontinuity across the shell. The equation above implies that a contribution proportional to the delta function in $\left\langle T_{u v}\right\rangle$ is necessary for the discontinuity of $\left\langle T_{u u}\right\rangle$. As the delta function is absent in the vacuum contribution to $\left\langle T_{u v}\right\rangle$ (3.14), the only chance for $\left\langle T_{u u}\right\rangle$ to be discontinuous is that the collapsing shell contributes a delta function term in $\left\langle T_{u v}\right\rangle$. Since the shell is collapsing at the speed of light, or equivalently, it lies on a null surface, the only component the shell can contribute to is $\left\langle T_{v v}\right\rangle$. Therefore, $\left\langle T_{u u}\right\rangle$ has to be continuous on the

\footnotetext{
${ }^{11}$ We need to take the higher-order corrections studied in Ref. [12] into consideration to derive this expression.
}

collapsing shell. ${ }^{12}$ Since the outgoing energy is zero in the flat spacetime inside the collapsing shell $\left(\left\langle T_{\text {ии }}\right\rangle=0\right.$ at $v=0^{-}$), we must have

$$
\left\langle T_{u и}\right\rangle=0
$$

just outside of the collapsing shell at $v=0^{+}$. In the next subsection, we will use this condition to determine the magnitude of Hawking radiation.

\section{Locus of collapsing shell}

As the areal radius of the shell $R_{s}$ is given by Eq. (3.6), its time-derivative is expressed as

$$
\dot{R}_{s}(u)=\dot{a}(u)-\frac{1}{8} e^{-\frac{u-u_{0}(u)}{2 a(u)}}+\frac{\kappa}{8 a(u)^{2}}+\mathcal{O}\left(\kappa^{2}\right),
$$

where we have chosen the position of the shell as $v=0$, and $u_{0}$ comes from the redefinition of $u$.

The time evolution of the Schwarzschild radius $\dot{a}(u)$ is related to the outgoing radiation via Eq. (3.8). According to Eq. (2.4), the outgoing energy flow $\left\langle T_{u u}\right\rangle$ at the neck is

$$
\left\langle T_{u u}\right\rangle=\frac{1}{R^{2}}\left(-\frac{1}{8 a^{2}(u)}+\beta(u)+\mathcal{O}(\kappa)\right) .
$$

The continuity condition of the outgoing energy flow across the collapsing shell fixes $\beta(u)$ as

$$
\beta(u)=\frac{1}{8 a^{2}(u)},
$$

so that Eqs. (3.8) and (3.19) give

$$
\dot{a}=-\frac{\kappa}{8 a^{2}}+\mathcal{O}\left(\kappa^{2}\right),
$$

and

$$
\dot{R}_{s}=-\frac{1}{8} e^{-\frac{u-u_{0}(u)}{2 a(u)}}+\mathcal{O}\left(\kappa^{2}\right) .
$$

The radius of the shell continues to decrease even after passing the neck (which is the local minimum of $R$ with respect to the variation in $x$ ). This is in fact necessary for the continuity of $R$ across the shell, as the areal radius of the incoming null surface must be monotonically decreasing from the viewpoint of an observer in the flat spacetime inside the shell. In terms of the $U$ coordinate in the flat spacetime, $R_{s}$ behaves as

$$
\partial_{U} R_{s}=-\frac{1}{2}
$$

\footnotetext{
${ }^{12}$ This is a consequence of the assumption of no pressure, i.e., $\left\langle T_{\theta \theta}\right\rangle=\left\langle T_{\phi \phi}\right\rangle=0$.
} 


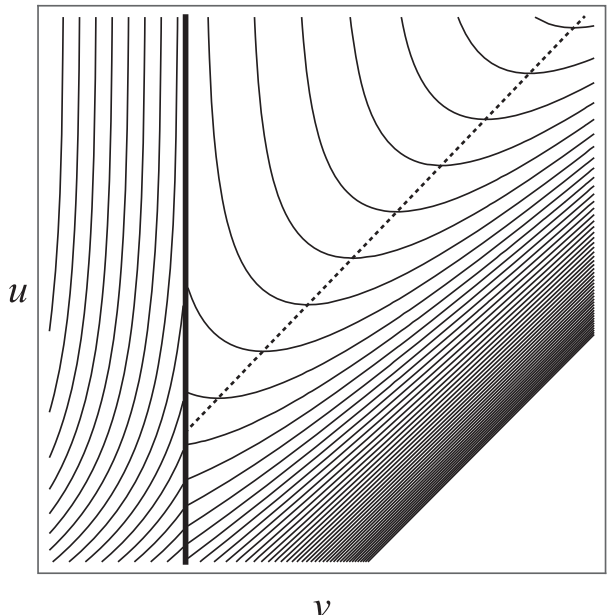

FIG. 2. Lines of $R(u, v)=$ const. in the geometry near the neck. Lines are just omitted in the lower-right of the figure since there are too many lines. The bold line is the collapsing thin shell. The dotted line is the neck, or equivalently, the apparent horizon. Lines on the upper side has smaller $R$ than those on the lower side since $\frac{\partial R}{\partial u}<0$. The lines on the right has larger $R$ than those on the left, namely $\frac{\partial R}{\partial v}>0$, except for the trapped region between the collapsing shell and the apparent horizon, where $\frac{\partial R}{\partial v}<0$.

which is consistent with the junction condition

$$
\frac{d U}{d u}=C=\frac{1}{4} e^{\left(x-x_{0}(u)\right) / a(u)} .
$$

It can be seen by comparing Eq. (3.23) with Eq. (3.22) that since $R_{s}(u)$ decreases at a rate that becomes exponentially small, the Schwarzschild radius $a(u)$ will decrease much faster than $R_{s}(u)$. Thus the difference $R_{s}(u)-a(u)$ is increasing while the radius $R_{s}(u)$ itself is decreasing.

In this section, we studied the geometry near the neck, the junction condition at the shell and their time evolution. The geometry is given by connecting the exterior geometry (3.5) and (3.6) with flat interior spacetime at the collapsing shell, $v=0$. The Schwarzschild radius $a(u)$ has the time evolution according to (3.22). The structure of the geometry is shown in Fig. 2. The geometry is consistent with that by Parentani and Piran, [5]. Thus the geometry of the evaporating black hole is interpreted as the time evolution of a space which has the structure of the "Wheeler's bag of gold."

It should be noted that our result in this section relies on the assumption that the time evolution of $x_{0}(u)$ is sufficiently small, $\dot{x}_{0}(u)=\mathcal{O}(\kappa)$. According to Eq. (3.23), the decrease in $R_{s}$ from $u_{1}$ to $u_{2}$ is

$$
\int_{u_{1}}^{u_{2}} d u \dot{R}_{S} \simeq \frac{a}{4}\left(e^{-\frac{u_{1}-u 0}{2 a}}-e^{\frac{u_{2}-u_{0}}{2 a}}\right)
$$

which is bounded from above by $a / 4$ as long as $u_{2}>$ $u_{1}>u_{0}$. This implies that the areal radius at the collapsing shell is still $\mathcal{O}(a)$ (while its decrease can be as large as $a / 4$ ) when the shell is still in the neighborhood (3.11) where the approximation is good. It should however be noted that the time evolution of $x_{0}(u)$ is assumed to be slow; $\dot{x}_{0}(u)=\mathcal{O}(\kappa)$. If instead $\dot{x}_{0}(u)=\mathcal{O}\left(\kappa^{0}\right)$, Eq. (3.23) becomes

$\dot{R}_{s}=-\frac{1}{8} e^{-\frac{u}{2 a(u)}}\left(1+2 \dot{x}_{0}(u)\right)+\frac{\kappa \dot{x}_{0}(u)}{4 a^{2}(u)}+\mathcal{O}\left(\kappa^{2}\right)$.

Thus, the discussion about $R_{s}(u)$ above depends on the assumption on $x_{0}(u)$. In order to check whether $\dot{x}_{0}(u)=$ $\mathcal{O}(\kappa)$, we should study the junction condition in the dynamical case in more details. In the next section, we consider the perturbative expansion around the outgoing Vaidya metric, which is valid for $r-a=\mathcal{O}(a)$ outside the apparent horizon, and show that the areal radius at the collapsing shell is still large as long as the shell is still in the neighborhood of the neck (3.11).

\section{BACKREACTION TO VAIDYA METRIC}

In the previous section, we have studied the black-hole geometry near the neck. In this section, we aim at connecting the geometry near the neck with the geometry in the asymptotically flat region.

To do so, we study the spacetime geometry in a different coordinate system, for which the most general spherically symmetric metric in $4 \mathrm{D}$ spacetime is of the form

$$
d s^{2}=-f(u, r) d u^{2}-2 d u d r+R^{2}(u, r) d \Omega^{2},
$$

where we have imposed the gauge-fixing condition $g_{u r}=g_{r u}=-1$. The geometry is assumed to be asymptotically flat, $f(u, r) \rightarrow 1$ and $R(u, r) \rightarrow r$ at the spatial infinity $r \rightarrow \infty$.

The coordinates in (4.1) is convenient for the outgoing Vaidya metric, which describes the geometry of the evaporating black hole around the asymptotic region, since only the Hawking radiation would be important in the quantum effects there. In order to solve the semiclassical Einstein equation for the black-hole geometry, we will consider the perturbation theory of the metric (4.1) starting with the outgoing Vaidya metric. Although the areal radius $R$ is commonly identified as the radial coordinate $r$, by imposing the gauge condition $R=r$ instead of $g_{u r}=g_{r u}=-1$, it is not very useful to study the backreaction to the Vaidya metric, because the areal radius $R$ has a local minimum and is thus not single-valued if the backreaction is taken into account. This is why we adopt another radial coordinate $r$ in the metric (4.1). As above, we assume that $\partial_{u} \sim \mathcal{O}(\kappa)$ in the $\kappa$-expansion.

As the expansion around the outgoing Vaidya metric is formally valid only for $r-a=\mathcal{O}(a)$ outside the Schwarzschild radius, we will consider another perturbation for $r-a=\mathcal{O}(\kappa)$ in order to check the validity of the 


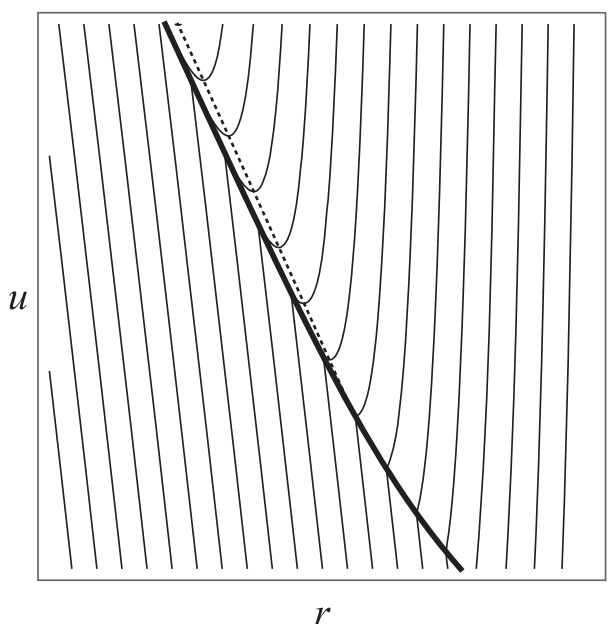

FIG. 3. Lines of $R(u, r)=$ const. in the $(u, r)$-coordinates. The bold line is the collapsing thin shell, and the dotted line is the neck, or equivalently, the apparent horizon. The distance between the collapsing shell and apparent horizon is very small in these coordinates. Strictly speaking, the distance in these coordinates is different from the proper distance, it can be a very rough estimate since $f(u, r)$ in (4.1) is small near the apparent horizon. More precise arguments are given in Sec. IV D and in Sec. V D.

result from the expansion around the outgoing Vaidya metric. ${ }^{13}$ We will see that the expansion around the outgoing Vaidya metric agrees with the result from another perturbative calculation for $r-a=\mathcal{O}(\kappa)$. We also find that the integration constants are related to the validity of the expansion at higher orders and should be chosen carefully. The advantage of this approach is that its description about the geometry around the neck is smoothly continued to the asymptotic region at large distance.

The main results of this section to be derived below are the following. The solution of the exterior geometry in the form of (4.1) is obtained as

$$
\begin{gathered}
f(u, r) \simeq 1-\frac{a(u)}{r}+\cdots, \\
R(u, r) \simeq r-\frac{\kappa}{4 a(u)} \log \left(\frac{r-a(u)+2 a(u) \dot{a}(u)}{a(u)}\right)+\cdots,
\end{gathered}
$$

where higher-order corrections indicated by "..." are of $\mathcal{O}(\kappa)$ for $r-a=\mathcal{O}(a)$ (far outside the apparent horizon) but $\mathcal{O}\left(\kappa^{2}\right)$ for $r-a=\mathcal{O}(\kappa)$ (around the apparent horizon). The geometry is given by connecting the solution above with the flat spacetime at the collapsing shell (See Fig. 3. The areal radius $R$ increases as it goes further inside the

\footnotetext{
${ }^{13}$ Here, we use the notation $y=\mathcal{O}(x)$ to indicate that $y$ is of the same order as $x$, but not less. For instance, it also implies that $y \gg \mathcal{O}\left(x^{2}\right)$ for $x \ll 1$.
}

apparent horizon $r=a(u)$ (as the value of $v$ gets smaller on a constant $-u$ curve), while it decreases along the collapsing shell (as the value of $u$ gets larger on a constant $v$ curve). The rate of decrease of the areal radius $R_{s}$ along the collapsing shell is much smaller than that of the Schwarzschild radius. We will see below that, although the difference in their areal radii $R$ will be as large as $\mathcal{O}(a)$, as the shell goes to deeper region inside the apparent horizon, the shell is only separated from the neck by a proper distance of the order of a Planck length.

While Eqs. (4.2) and (4.3) are not very useful deep inside the neck where $r-a=\mathcal{O}(a)$, we will study the collapsing shell deep inside the neck in more detail in Sec. V.

\section{A. Perturbation of Vaidya metric}

We first take into account the effect of the Hawking radiation by introducing the outgoing energy at the leading order of the expansion, so that the solution of the semiclassical Einstein equation at the leading order is given by the outgoing Vaidya metric. Then we treat the remaining part of the vacuum energy-momentum tensor (including the incoming negative vacuum energy flux) as a perturbation.

The energy-momentum tensor (2.4)-(2.7) is fixed by the trace anomaly and the conservation law. For the metric (4.1), it is equivalent to

$$
\begin{gathered}
\left\langle T_{u u}\right\rangle=\frac{1}{R^{2}}\left[\beta+\gamma+f \partial_{r}^{2} f-\frac{1}{4}\left(\partial_{r} f\right)^{2}\right]+\mathcal{O}(\kappa), \\
\left\langle T_{r r}\right\rangle=\frac{1}{R^{2} f^{2}}\left[2 \gamma+f \partial_{r}^{2} f-\frac{1}{2}\left(\partial_{r} f\right)^{2}\right]+\mathcal{O}(\kappa), \\
\left\langle T_{u r}\right\rangle=\left\langle T_{r u}\right\rangle=\frac{1}{R^{2} f}\left[\gamma+f \partial_{r}^{2} f-\frac{1}{4}\left(\partial_{r} f\right)^{2}\right]+\mathcal{O}(\kappa) .
\end{gathered}
$$

We solve the semiclassical Einstein equation order by order in the $\kappa$-expansion. We expand $f(u, r)$ and $R(u, r)$ as

$$
\begin{aligned}
& f(u, r)=f_{0}(u, r)+\kappa f_{1}(u, r)+\kappa^{2} f_{2}(u, r)+\mathcal{O}\left(\kappa^{3}\right), \\
& R(u, r)=R_{0}(u, r)+\kappa R_{1}(u, r)+\kappa^{2} R_{2}(u, r)+\mathcal{O}\left(\kappa^{3}\right) .
\end{aligned}
$$

At the leading order, the semiclassical Einstein equation is solved by

$$
\begin{aligned}
& R_{0}(u, r)=R_{00}(u)+R_{01}(u) r, \\
& f_{0}(u, r)=R_{01}^{-2}(u)-\frac{a(u)}{R_{0}(u, r)},
\end{aligned}
$$

where $R_{00}(u), R_{01}(u)$, and $a(u)$ are integration constants.

In the lowest-order approximation, $a(u)$ corresponds to the Schwarzschild radius. The other integration constants $R_{00}(u)$ and $R_{01}(u)$ can be absorbed by the redefinition of the $(u, r)$-coordinates. Note that $u$-dependence is very slow 
and contribute only to the higher-order corrections, for example, $\dot{a}(u)=\mathcal{O}(\kappa)$. We choose the gauge condition $R_{00}(u)=0$ and $R_{01}(u)=1$ to obtain the standard expression of the outgoing Vaidya metric;

$$
\begin{gathered}
R_{0}(u, r)=r, \\
f_{0}(u, r)=1-\frac{a(u)}{r} .
\end{gathered}
$$

At the next-to-leading order, the semiclassical Einstein equation is solved by

$$
\begin{aligned}
R_{1}(u, r)= & R_{10}(u)+R_{11}(u) r \\
& +\frac{1}{8 r a^{2}(u)}\left[a(u)^{2}\left(8 \gamma(u) r^{2}-3\right)\right. \\
& -2 r(r-2 a(u)) \log (r) \\
& \left.-2 r(r-2 a(u))\left(4 \gamma(u) a(u)^{2}-1\right) \log (r-a(u))\right],
\end{aligned}
$$

$$
\begin{aligned}
f_{1}(u, r)= & \frac{a_{1}(u)}{r}+\frac{1}{2 r^{3} a^{2}(u)}\left[a^{2}(u)\left(r-a(u)-2 r^{3} \gamma(u)\right)\right. \\
& +2 r a(u)^{2}\left(a(u) R_{10}(u)-2 r^{2} R_{11}(u)\right) \\
& +\left(-2 r^{2} a(u)+r a(u)^{2}+r^{3}\right) \log r \\
& \left.+r(r-a(u))^{2}\left(4 a(u)^{2} \gamma(u)-1\right) \log (r-a(u))\right],
\end{aligned}
$$

$$
\dot{a}(u)=-\kappa \beta(u),
$$

where $R_{10}(u), R_{11}(u)$, and $a_{1}(u)$ are the integration constants, which will be set to 0's as they can be absorbed by the parameters $R_{00}(u), R_{01}(u)$, and $a(u)$ at the leading order, respectively.

The first-order solution behaves in the asymptotic region $(r \rightarrow \infty)$ as

$$
\begin{gathered}
R_{1}(u, r)=\gamma(r-r \log r)+\mathcal{O}\left(r^{0}\right), \\
f_{1}(u, r)=-\gamma(1-2 \log r)+\mathcal{O}\left(r^{-1}\right) .
\end{gathered}
$$

By imposing $\gamma=0$ (3.1), which corresponds to the condition of no incoming energy in the past null infinity, Eqs. (4.13) and (4.14) are smaller than the leading-order solutions Eqs. (4.11) and (4.12), respectively, and hence the perturbative expansion is valid for large $r$.

On the other hand, the first-order corrections (4.13) and (4.14) behave near the Schwarzschild radius $r=a(u)$ as

$R_{1}(u, r)=-\frac{1}{8 a(u)}\left[3+2 \log \left(\frac{r-a(u)}{a(u)}\right)\right]+\mathcal{O}(r-a(u))$,

$$
f_{1}(u, r)=\frac{r-a(u)}{2 a(u)^{3}}+\mathcal{O}\left((r-a(u))^{2}\right) .
$$

For $f(u, r)$, the first-order correction is smaller than the leading-order solution and the expansion is valid even around $r=a$. The negative vacuum energy introduces a small correction:

$$
\begin{aligned}
f(u, r) & =f_{0}(u, r)+f_{1}(u, r)+\cdots \\
& =\left(\frac{1}{a(u)}+\frac{\kappa}{2 a^{3}(u)}\right)(r-a(u))+\mathcal{O}\left((r-a(u))^{2}\right) .
\end{aligned}
$$

However, the correction term $R_{1}(u, r)$ for the radius $R(u, r)$ has a logarithmic divergence at $r=a(u)$.

The areal radius $R(u, r)$ has a local minimum slightly outside the point $r=a(u)$ where $R_{1}(u, r)$ approaches positive infinity. The local minimum of $R$ (the neck) is located at the point where

$$
0=\partial_{r} R(u, r) \simeq 1-\frac{\kappa}{4 a(u)[r-a(u)]}+\cdots,
$$

which is solved by

$$
r=a(u)+\frac{\kappa}{4 a(u)}+\mathcal{O}\left(\kappa^{2}\right) .
$$

This is consistent with the structure we have seen in previous sections.

Now, we consider a collapsing shell at the speed of light. The shell is located on an incoming null line $r=r_{s}(u)$, which is a solution of the differential equation

$$
\frac{d r_{s}}{d u}=-\frac{1}{2} f\left(u, r_{s}\right)
$$

When the shell approaches the Schwarzschild radius $\left(r_{s}-a(u)=\mathcal{O}(\kappa)\right)$, the position of the shell $r_{s}(u)$ is approximately given by

$$
r_{s}(u)=a(u)+s e^{-\frac{u}{2 a(u)}}-2 a(u) \dot{a}(u)+\mathcal{O}\left(\kappa^{2}\right),
$$

where $s$ is an integration constant. Recall that the time evolution of $a(u)$ is slow; $\dot{a}(u)=\mathcal{O}(\kappa)$. As the second term is exponentially suppressed over time, the shell approaches

$$
r \simeq a(u)-2 a(u) \dot{a}(u)+\mathcal{O}\left(\kappa^{2}\right) .
$$

This is slightly outside the point $r=a(u)$ as $a(u)$ is decreasing. The shell never reaches the value in Eq. (4.25) within finite time, unless $s=0$ in Eq. (4.24). The null 
surface (4.25) can be interpreted as the past horizon of the exterior geometry. ${ }^{14}$

The Hawking radiation $\beta(u)$ can be calculated from the junction condition on the collapsing shell. The condition is given by $\left\langle T_{u u}\right\rangle=0$ in $(u, v)$-coordinates, that is,

$$
\begin{aligned}
0 & =\left\langle T_{u u}(u, v)\right\rangle \\
& =\left\langle T_{u u}\left(u, r_{s}\right)\right\rangle+2\left(\frac{\partial r}{\partial u}\right)\left\langle T_{u r}\left(u, r_{s}\right)\right\rangle+\left(\frac{\partial r}{\partial u}\right)^{2}\left\langle T_{r r}\left(u, r_{s}\right)\right\rangle \\
& =\beta(u)+\frac{a\left(3 a-4 r_{s}\right)}{8 r_{s}^{4}}+\mathcal{O}(\kappa) .
\end{aligned}
$$

As Eq. (4.25) implies that $r_{s}-a(u)=\mathcal{O}(\kappa)$, the Hawking radiation is estimated as

$$
\beta(u)=\frac{1}{8 a^{2}(u)}+\mathcal{O}(\kappa),
$$

in agreement with Eq. (3.21). Thus, the time derivative of the Schwarzschild radius $\dot{a}(u)$ is given by (4.15)

$$
\dot{a}(u)=-\kappa \beta(u)=-\frac{\kappa}{8 a^{2}(u)} .
$$

According to Eq. (4.25), the position of the shell approaches

$$
r_{s}=a(u)+\frac{\kappa}{4 a(u)} .
$$

This is nothing but the $r$-coordinate (4.22) of the neck.

Naively, this result seems to imply that the $r$-coordinate at the collapsing shell differs from that at the apparent horizon only by $\mathcal{O}\left(\kappa^{2}\right)$ after it gets inside the neck. However, this naive expectation is incorrect for our perturbative expansion. To find more precisely the relative position between the collapsing shell and the apparent horizon, we have to include the second-order effect. In the next subsection, we will study the second-order correction to argue that the difference between the areal radius at the shell and that at the apparent horizon at the same $u$ increases with $u$.

\section{B. Higher-order correction}

In the perturbative expansion around the Vaidya metric (4.1), the first-order correction of the areal radius $R$ is not much smaller than the leading order term in the region where $r-a \sim \mathcal{O}(\kappa)$. In addition, another issue with the first-order approximation is the following. The divergence

\footnotetext{
${ }^{14}$ The black-hole geometry for a collapsing null shell is obtained by connecting this exterior geometry with flat spacetime on the collapsing shell with $s>0$. The past horizon (4.25) does not really exist since this region of the exterior geometry is replaced by the flat spacetime.
}

of the first-order correction to the areal radius $R$ at $r=a$ looks harmless as it is located inside the (nonexistent) past horizon, hence outside the region of interest. This is because the past horizon is moved by the firstorder correction slightly outward to $r=a-2 a \dot{a}>a$. However, this difference is only of $\mathcal{O}(\kappa)$, and the position of the divergence in $R$ can be read off from Eq. (4.13) only up to an $\mathcal{O}(\kappa)$-correction, so the statement above about the divergence is not reliable. To confirm the relative position of the divergence in $R$ with respect to the past horizon, the second-order correction must be studied.

In this subsection, we investigate the second-order correction. The second-order correction to $R$ turns out to have a pole at $r=a$, which is comparable to the first order term, and naively implies the breakdown of the perturbative expansion. However, we can absorb the pole in the secondorder term by a shift of the logarithmic divergence in the 1st-order term. This implies that the pole at the second order can be canceled by using an appropriate redefinition of the coordinate $r$, or equivalently, by choosing integration constants at the first order appropriately.

This is in fact related to the relative position between the past horizon (4.25) and the logarithmic divergence of $R$ in Eq. (4.13). The relative position at $\mathcal{O}(\kappa)$ is related to the validity of the perturbative expansion at the second order. As the leading-order term of the difference between the $r$-coordinates of the collapsing shell and the apparent horizon is $\mathcal{O}(\kappa)$, the second-order correction is important to remove the ambiguity of the position at $\mathcal{O}(\kappa)$. The thirdorder correction should be taken into consideration to calculate the $\mathcal{O}\left(\kappa^{2}\right)$-correction to the positions, etc. To justify the assumption that the divergence in higher-order terms can be canceled by using an appropriate definition of the $r$-coordinate, we will consider another perturbative expansion in Sec. IV C. In the end, the second-order result in this subsection allows us to smoothly connect the solution of the metric in the asymptotically flat region to the near-horizon region.

The expectation value of the energy-momentum tensor is expanded as

$$
\left\langle T_{\mu \nu}\right\rangle=T_{\mu \nu}^{(0)}+\kappa T_{\mu \nu}^{(1)}+\mathcal{O}\left(\kappa^{2}\right) .
$$

The leading-order terms $T_{\mu \nu}^{(0)}$ are given by (4.4)-(4.6), in which the $u$-derivatives are ignored as higher-order terms. The linear-order corrections $T_{\mu \nu}^{(1)}$ contains the effects of $u$ derivatives of leading-order terms. By using the solution at the leading order (4.11)-(4.12), $T_{\mu \nu}^{(1)}$ is calculated via the conservation law at the first order of the $\kappa$-expansion as

$$
\begin{aligned}
T_{u u}^{(1)}= & \beta_{1}(u)+\frac{a(u) \dot{a}(u)}{2 \kappa r^{2}(r-a(u))}-\frac{2 a(u)}{r^{3}} f_{1}(u, r) \\
& -\frac{a(u)}{2 r^{2}} \partial_{r} f_{1}(u, r)\left(1-\frac{a(u)}{r}\right) \partial_{r}^{2} f_{1}(u, r),
\end{aligned}
$$




$$
\begin{aligned}
T_{r r}^{(1)}= & \frac{r}{r-a(u)} \gamma_{1}(u)+\frac{2 r \dot{a}(u)}{\kappa(r-a(u))^{3}} \\
& +\frac{a(u)(2 r-a(u))}{r(r-a(u))^{3}} f_{1}(u, r)-\frac{a(u)}{(r-a(u))^{2}} \partial_{r} f_{1}(u, r) \\
& +\frac{r}{r-a(u)} \partial_{r}^{2} f_{1}(u, r),
\end{aligned}
$$

$$
\begin{aligned}
T_{u r}^{(1)}= & T_{r u}^{(1)}=\frac{2 r^{2}}{(r-a(u))^{2}} \gamma_{1}(u)+\frac{\dot{a}(u)}{\kappa(r-a(u))^{2}} \\
& +\frac{a^{2}(u)}{4 r^{2}(r-a(u))^{2}} f_{1}(u, r)-\frac{a(u)}{2 r(r-a(u))^{2}} \partial_{r} f_{1}(u, r) \\
& +\partial_{r}^{2} f_{1}(u, r),
\end{aligned}
$$

where $\beta_{1}$ and $\gamma_{1}$ are integration constants. The integration constant $\beta_{1}$ corresponds to the linear-order correction to the Hawking radiation. The other constant $\gamma_{1}$ is the linear-order correction to the incoming energy at the past infinity; we should choose $\gamma_{1}=0$.

Solving the semiclassical Einstein equation at the second order, there is a divergence in the second-order term of $R$. It is

$$
R_{2}(u, r)=-\frac{\dot{a}(u)}{2 \kappa(r-a(u))}+\mathcal{O}\left((r-a(u))^{0}\right),
$$

which is large compared with the first-order correction to the areal radius $R$ if $r-a(u)=\mathcal{O}(\kappa)$. Together with the logarithmic term in $R_{1}(u, r)$,

$$
R_{1}(u, r)=-\frac{1}{4 a(u)} \log (r-a(u))+\cdots,
$$

the pole in $R_{2}(u, r)$ amounts to a shift of the location of the logarithmic divergence in $R_{1}(u, r)$ as

$$
\begin{aligned}
- & \frac{1}{4 a(u)} \log (r-a(u))-\frac{\dot{a}(u)}{2(r-a(u))} \\
& \left.\simeq-\frac{1}{4 a(u)} \log (r-a(u)+2 a(u) \dot{a}(u))\right) .
\end{aligned}
$$

Although the pole in Eq. (4.34) can be interpreted as a small shift of the logarithmic divergence in $R_{1}(u, r)$, it seemingly breaks down the perturbative expansion since it becomes too large as a second-order correction. This interpretation above of the 2nd-order term (4.34) can be justified more precisely as follows. To avoid the divergence in $R_{2}(u, r)$, we need to keep the integration constant $R_{10}(u)$ in Eq. (4.13) as an arbitrary function of $u$, and then $R_{2}(u, r)$ would be found to be

$$
R_{2}(u, \tilde{r})=-\frac{R_{10}(u)+2 a(u) \dot{a}(u)}{4 a(u) \kappa(\tilde{r}-a(u))}+\mathcal{O}\left((\tilde{r}-a(u))^{0}\right),
$$

where we denoted the $r$-coordinate as $\tilde{r}$, in order to distinguish from the $r$-coordinate used in the $R_{10}(u)=0$ gauge. Different choices of $R_{10}(u)$ corresponds to different definitions of the $r$-coordinates which are related to each other by coordinate transformations, $r \rightarrow \tilde{r}=r-R_{10}(u)$. The integration constant $R_{10}(u)$ should be chosen such that the pole in (4.37) cancels;

$$
R_{10}(u)=-2 a(u) \dot{a}(u) .
$$

Then, the second-order correction is negligible and the perturbation is valid. By using (4.38), or equivalently $\tilde{r}$, the position of the logarithmic divergence is not shifted away from $\tilde{r}=a(u)$. Since the $r$-coordinate in the $R_{10}(u)=0$ gauge is related to $\tilde{r}$, the $r$-coordinate in the gauge (4.38), via the relation $r=\tilde{r}+R_{10}(u)$, the position of the divergence is indeed shifted to

$$
r=a(u)-2 a(u) \dot{a}(u) .
$$

On the other hand, $f(u, r)$ does not have the divergence in the second-order correction. By using Eq. (4.38), instead of $R_{10}(u)=0$, Eq. (4.14) gives $\mathcal{O}(\kappa)$ correction to $f(u, \tilde{r})$ as

$$
f(u, \tilde{r})=\tilde{r}-a(u)-2 a(u) \dot{a}(u)+\mathcal{O}\left(\kappa^{2}\right),
$$

for $\tilde{r}-a(u)=\mathcal{O}(\kappa)$, which is equivalent to $f(u, r)=$ $r-a(u)+\mathcal{O}\left(\kappa^{2}\right)$ after the coordinate transformation to the $R_{10}(u)=0$ gauge.

Similarly, the position of the past horizon of the exterior geometry is placed at the same position (4.25), up to $\mathcal{O}\left(\kappa^{2}\right)$. The local minimum of the radius $R$ is now placed at

$$
r=a(u)-2 a(u) \dot{a}(u)+\frac{\kappa}{4 a(u)},
$$

as a second-order correction to Eq. (4.22).

In this subsection, we have seen that the $r$-coordinate should be chosen appropriately for the validity of the perturbation. The solutions (4.2) and (4.3) can be calculated at the first order with an appropriate choice of the integration constant, while the appropriate choice is found by studying the second-order terms. Since the second-order corrections are important to see the relative position of the past horizon and the logarithmic divergence of the areal radius to $\mathcal{O}(\kappa)$ corrections, we expect that the third-order corrections would be necessary only for $\mathcal{O}\left(\kappa^{2}\right)$ correction to the relative position. However, as there can be higher-order divergences in higher-order terms, it is not totally obvious that all higher-order corrections will never introduce a large correction to the first-order solution at $r-a=\mathcal{O}(\kappa)$ in the perturbative expansion around the Vaidya metric.

In the next subsection, we consider another expansion focused on the neighborhood of $r-a=\mathcal{O}(\kappa)$, and justify our claim that the corrections to the second order are 
sufficient to describe the geometry near and outside the apparent horizon, so that the Vaidya-like metric (4.1) allows us to describe the geometry in the near-horizon region $r-a=\mathcal{O}(\kappa)$ as well as in the asymptotically flat region with good approximation.

\section{Perturbation near the neck}

In the perturbative expansion around the Vaidya metric discussed above, it is implicitly assumed that $r-a(u) \sim$ $\mathcal{O}\left(\kappa^{0}\right)$, and it is not totally clear if the expansion is valid for the smaller neighborhood where $r-a(u) \sim \mathcal{O}(\kappa)$, as we have mentioned above. To clarify the subtlety involved, we consider another perturbative expansion which is good around the local minimum of $R$, and show that the results in the previous subsection is indeed valid in this region.

Instead of Eq. (4.1), we consider the following metric:

$$
d s^{2}=-\tilde{f}(u, z) d u^{2}-2 \kappa d u d z+R^{2}(u, z) d \Omega^{2} .
$$

Here, we focus on the small neighborhood around the neck. We identify the radial coordinate $z$ with the $r$-coordinate in Eq. (4.1) via

$$
r=a(u)+\kappa z,
$$

and so $\tilde{f}(u, z)$ is related to $f(u, r)$ in Eq. (4.1) as

$$
\tilde{f}(u, z)=f(u, r)+2 \dot{a}(u) .
$$

Around $r=a(u)$, we have

$$
\tilde{f}(u, z)=\mathcal{O}(\kappa) .
$$

At the neck,

$$
R(u, z)=a(u)+\mathcal{O}(\kappa) .
$$

We consider the following expansion:

$$
\begin{gathered}
\tilde{f}(u, z)=\kappa \tilde{f}_{0}(u, z)+\mathcal{O}\left(\kappa^{2}\right), \\
R(u, z)=a(u)+\kappa \tilde{R}_{0}(u, z)+\mathcal{O}\left(\kappa^{2}\right) .
\end{gathered}
$$

The leading-order terms of the semiclassical Einstein equation give the following differential equations for $\tilde{f}_{0}(u, z)$ and $\tilde{R}_{0}(u, z)$;

$$
\begin{aligned}
0= & \left(\partial_{z} \tilde{f}_{0}(u, z)\right)^{2}-2 \tilde{f}_{0}(u, z)\left(\partial_{z}^{2} \tilde{f}_{0}(u, z)\right. \\
& \left.+2 a(u) \tilde{f}_{0}(u, z) \partial_{z}^{2} \tilde{R}_{0}(u, z)\right), \\
0= & \left(\partial_{z} \tilde{f}_{0}(u, z)\right)^{2}-4 \tilde{f}_{0}(u, z) \\
& \times\left[1-a(u)\left(\partial_{z} \tilde{f}_{0}(u, z)\right)\left(\partial_{z} \tilde{R}_{0}(u, z)\right)\right],
\end{aligned}
$$

$$
0=\kappa \beta(u)+a(u) \dot{a}(u) \partial_{z} \tilde{f}_{0}(u, z),
$$

where we have again assumed that the $u$-derivative is small: $\partial_{u} \sim \mathcal{O}(\kappa)$.

One of the solutions of the differential equations above is trivial-the first-order correction of $R$ is constant in the radial direction, $\tilde{R}_{0}(u, z)=\tilde{R}_{0}(u)$. The other nontrivial solution is

$$
\begin{gathered}
\tilde{f}_{0}(u, z)=\tilde{f}_{00}(u)+\tilde{f}_{01}(u) z \\
\tilde{R}_{0}(u, z)=\tilde{R}_{00}(u)+\frac{z}{a(u) f_{01}(u)}-\frac{1}{4 a(u)} \log \tilde{f}_{0}(u, z),
\end{gathered}
$$

where $\tilde{f}_{00}(u), \tilde{f}_{01}(u)$ and $\tilde{R}_{00}(u)$ are arbitrary functions. This result is consistent with the expansion around the Vaidya metric, Eqs. (4.2)-(4.3). By using Eqs. (4.43) and (4.44), Eqs. (4.2) and (4.3) give the same expressions as Eqs. (4.52) and (4.53). The integration constants are fixed by the relation to Eqs. (4.2) and (4.3) as

$$
\begin{gathered}
\tilde{f}_{0}(u, z)=2 \kappa^{-1} \dot{a}(u)+\frac{z}{a(u)}, \\
\tilde{R}_{0}(u, z)=-\frac{3}{8 a(u)}+z-\frac{1}{4 a(u)} \log \left(2 \kappa^{-1} \dot{a}(u)+\frac{z}{a(u)}\right) .
\end{gathered}
$$

Equation (4.51) implies that the Hawking radiation satisfies

$$
\dot{a}(u)=-\kappa \beta(u) .
$$

Therefore, we have verified that the expressions (4.2)-(4.3) are indeed reliable even near the apparent horizon $r-a=\mathcal{O}(\kappa)$.

Now we consider the time-evolution of the collapsing shell using Eqs. (4.2) and (4.3). Since the higher-order corrections to $f(u, r)$ is negligible even for $r-a=\mathcal{O}(\kappa)$, the locus of the collapsing shell is given by Eq. (4.24), and the areal radius $R$ there is calculated as

$$
\begin{aligned}
R_{s}(u) & \simeq r_{s}(u)-\frac{\kappa}{4 a(u)} \log \left(\frac{r_{s}(u)-a(u)+2 a(u) \dot{a}(u)}{a(u)}\right)+\cdots \\
& \simeq a(u)+s e^{-\frac{u}{2 a(u)}}+\frac{\kappa u}{8 a^{2}(u)}+\cdots,
\end{aligned}
$$

whose time evolution is

$$
\dot{R}_{s}(u)=\dot{a}(u)-\frac{s}{2 a(u)} e^{-\frac{u}{2 a(u)}}+\frac{\kappa}{4 a^{2}(u)}+\mathcal{O}\left(\kappa^{2}\right)
$$




$$
=-\frac{s}{2 a(u)} e^{-\frac{u}{2 a(u)}}+\mathcal{O}\left(\kappa^{2}\right) .
$$

Since $s>0$, the areal radius at the shell is always decreasing with time.

\section{Validity of perturbation}

So far, we calculated the solution of the semiclassical Einstein equation for the Unruh vacuum, perturbatively. We first assumed that the radius is not very close to the Schwarzschild radius, $r-a=\mathcal{O}(a)$. The resulting perturbative series of the metric components $f(u, r)$ and $R(u, r)$ would be valid even near the apparent horizon $r-a=$ $\mathcal{O}(\kappa)$ since the higher-order terms are further suppressed than the lower-order terms, for example, $f_{0} / f_{1} \gg \kappa$. It turns out that it agrees with the result of another $\kappa$-expansion for $r-a=\mathcal{O}(\kappa)$. Although it is difficult to show that the perturbative series converge, our result at least gives a good approximation for $r-a=\mathcal{O}(\kappa)$.

In order to find out the range of validity of the perturbative expansion, we examine the expansion of some geometric quantities. By using our results of the metric components $f$ and $R$, it is straightforward to calculate the Ricci scalar $\mathcal{R}$, Ricci tensor $\mathcal{R}_{\mu \nu}$ and Riemann tensor $\mathcal{R}_{\mu \nu \rho \sigma}$. Some scalar quantities are evaluated as

$$
\begin{gathered}
\mathcal{R}=-\frac{2 \kappa a(u)}{r^{5}}+\mathcal{O}\left(\kappa^{2}\right), \\
\mathcal{R}^{\mu \nu} \mathcal{R}_{\mu \nu}=\mathcal{O}\left(\kappa^{2}\right),
\end{gathered}
$$

$$
\begin{aligned}
\mathcal{R}^{\mu \nu \rho \sigma} \mathcal{R}_{\mu \nu \rho \sigma} & \\
= & \frac{12 a^{2}(u)}{r^{6}}+\frac{12 \kappa}{r^{6}}\left(\log \frac{r-a(u)}{r}+\mathcal{O}\left((r-a(u))^{0}\right)\right) \\
& +\frac{144 a^{3}(u) \dot{a}(u)}{r^{7}}+\mathcal{O}\left(\kappa^{2}\right)
\end{aligned}
$$

Thus the perturbative expansion would be valid for

$$
r-a \gg a e^{-a^{2} / \kappa},
$$

as long as $\dot{a}(u)=-\kappa \beta(u)=\mathcal{O}(\kappa)$. (Notice that the second term on the right-hand side of Eq. (4.63) is $\sim \kappa \log \kappa$ for $r-a=\mathcal{O}(\kappa) \gg a e^{-a^{2} / \kappa}$, which vanishes in the limit $\kappa \rightarrow 0$.) On the other hand, around the end of the evaporation, when the Schwarzschild radius approaches to the Planck length, the Hawking radiation becomes very strong as $\beta(u)=\frac{1}{8 a^{2}(u)}=\mathcal{O}\left(\kappa^{-1}\right)$, and then, the perturbative expansion breaks down.

\section{Distance between apparent horizon and collapsing shell}

In this subsection, we calculate more precisely the distance from the collapsing shell to the apparent horizon. In order to estimate the distance, we consider geodesics between the collapsing shell and the apparent horizon.

In the previous sections, we have seen that the quantum corrections become important in $R$ but are negligible in $f$, in the metric (4.1). So, $f$ is simply given by Eq. (4.2) up to the corrections of $\mathcal{O}\left(\kappa^{2}\right)$. We expect that the higher-order corrections of $f$ would not give divergences around $r=a(u)$, unlike the areal radius $R$. Thus, naive perturbation around the Vaidya metric implies that the expression (4.2) would be good everywhere $r \geq a(u)$, although the perturbation breaks down due to the divergence in $R$. Here, we focus on the 2-dimensional part of $(u, r)$-directions. Since $R$ is irrelevant for this 2-dimensional part, we assume that Eq. (4.2) even holds deep inside the neck, and consider $r-a \leq \mathcal{O}(\kappa)$ (including $r-a \ll \kappa / a) .{ }^{15}$

It is convenient to introduce a new coordinate $\chi$ as

$$
r=a(u)-2 a(u) \dot{a}(u)+\frac{\kappa}{4 a(u)} \chi^{2},
$$

or equivalently,

$$
z=-2 \kappa^{-1} a(u) \dot{a}(u)+\frac{\chi^{2}}{4 a(u)},
$$

with $\chi=\mathcal{O}(1)$. Since we assume that variation of the Schwarzschild radius with time is very slow $(\dot{a}(u)=\mathcal{O}(\kappa))$, $a(u)$ can be treated as a constant in the domain of the approximation (3.11).

The (2-dimensional part of) the metric is expressed as

$$
d s^{2}=-\kappa \frac{\chi^{2}}{4 a^{2}} d u^{2}-\kappa \frac{\chi}{a} d \chi d u .
$$

We define the $\tau$-coordinate as

$$
d u=d \tau-2 a \frac{d \chi}{\chi},
$$

and the metric becomes

$$
d s^{2}=\frac{\kappa}{4 a^{2}}\left(-\chi^{2} d \tau^{2}+4 a^{2} d \chi^{2}\right) .
$$

This is nothing but the Rindler space.

In the original $r$-coordinate, the past horizon is located at

$$
r=a(u)-2 a(u) \dot{a}(u) .
$$

\footnotetext{
${ }^{15}$ The divergence in $R$ may affect the validity of the expression (4.2) deep inside the neck. We will study the region deep inside the neck in the next section. The conclusion remains the same.
} 
The collapsing shell is hence always located outside the past horizon. ${ }^{16}$ The apparent horizon is located at

$$
r=a(u)-2 a(u) \dot{a}(u)+\frac{\kappa}{4 a(u)},
$$

which corresponds to $\chi=1$.

To calculate the proper distance, it is convenient to use the coordinates of the flat spacetime;

$$
T=\chi \sinh (\tau / 2 a), \quad X=\chi \cosh (\tau / 2 a),
$$

in terms of which the metric is

$$
d s^{2}=\kappa\left(-d T^{2}+d X^{2}\right) .
$$

The apparent horizon, or equivalently the $\chi=1$ line, is a hyperbolic curve $-T^{2}+X^{2}=1$. The distance between the origin $X=T=0$ to the apparent horizon $\chi=1$ is always 1 in the Minkowski space up to the factor $\sqrt{\frac{\kappa}{4 a^{2}}}$. Since the collapsing shell is located on the future side of the past horizon $X+T=0$, the distance from any point on the collapsing shell to the apparent horizon before the shell crosses the future horizon is always smaller than that from the origin. Thus the distance is smaller than $\ell_{p} \equiv \kappa^{1 / 2}$.

The calculation above about the Planckian distance between the collapsing shell and the apparent horizon is based on the metric derived for the near-horizon region. (Furthermore, we have ignored the time-dependence by dropping $\dot{a}$.) A priori it does not have to hold when the shell is deep inside the horizon. More discussions on the scale of this proper distance deep inside the horizon will be given in the next section.

\section{DEEP INSIDE THE NECK}

So far, we have studied the spacetime geometry from the asymptotic region to the near-horizon region, including a small neighborhood slightly inside the apparent horizon where $R-a=\mathcal{O}(\kappa / a)$. In this section, we shall study the geometry deeper inside the apparent horizon where $R-a=\mathcal{O}(a)$.

Note that the phrase "deep inside the neck" in this paper does not imply a large proper distance in the radial direction between the apparent horizon and a point in this region. It merely refers to a region inside the neck where the areal radius $R$ is significantly larger than its value at the neck.

\section{A. Dynamical geometry deep inside the neck}

In this subsection, we study the dynamical geometry deep inside the neck in the sense that $R-a$ is of order

\footnotetext{
${ }^{16}$ The spacetime geometry (4.69) under consideration only applies to the exterior space of the collapsing shell. (The interior space is flat spacetime.) Hence the past horizon actually does not exist.
}

$\mathcal{O}\left(\kappa^{0}\right)$, for arbitrary $\beta$ and $\gamma$ in the semiclassical Einstein equations (2.4) and (2.5). We will determine the values of $\beta$ and $\gamma$ in the next subsection for a collapsing null shell.

According to Eq. (3.5), the red-shift factor $C$ becomes exponentially small when $x_{0}-x \gg a(u)$. Therefore, in the region deep inside the neck where Eq. (2.26) holds, higherorder terms in the $C$-expansion can be ignored. With this approximation, the semiclassical Einstein equation is approximated by the following equations:

$$
\begin{gathered}
0=-2 \kappa \beta R^{2}+\left(\partial_{u} R^{2}\right)^{2}+4 R^{2}\left(\partial_{u} R^{2}\right) \partial_{u} \rho \\
-2 R^{2}\left[\left(\partial_{u} R^{2}\right)^{2}-2 \kappa\left(\partial_{u} \rho\right)^{2}+2 \kappa \partial_{u}^{2} \rho\right], \\
0=-2 \kappa \gamma R^{2}+\left(\partial_{v} R^{2}\right)^{2}+4 R^{2}\left(\partial_{v} R^{2}\right) \partial_{v} \rho \\
-2 R^{2}\left[\left(\partial_{v} R^{2}\right)^{2}-2 \kappa\left(\partial_{v} \rho\right)^{2}+2 \kappa \partial_{v}^{2} \rho\right], \\
0 \simeq \partial_{u} \partial_{v} R^{2}+2 \kappa \partial_{u} \partial_{v} \rho .
\end{gathered}
$$

The first two equations (5.1) and (5.2) are exactly equivalent to $G_{u u}=\kappa\left\langle T_{u u}\right\rangle$ and $G_{v v}=\kappa\left\langle T_{v v}\right\rangle$. The third equation (5.3) is equivalent to $G_{u v}=\kappa\left\langle T_{u v}\right\rangle$ up to the term $C / 2$, which can be neglected for small $C$.

From Eq. (5.3), we obtain

$$
\rho \simeq-\frac{R^{2}}{2 \kappa}+F(v)+\bar{F}(u),
$$

where $F(v)$ and $\bar{F}(u)$ are the integration constants.

Remarkably, the other two nonlinear differential equations (5.1), (5.2) can be solved exactly (to all orders in $\kappa$ ) by using Eq. (5.4) as

$\sqrt{R^{2}\left(R^{2}-\kappa\right)}-\alpha \log \left(R+\sqrt{R^{2}-\kappa}\right)=G(v)+\bar{G}(u)$,

where $G(v)$ and $\bar{G}(u)$ are given by

$G(v)= \pm 2 \kappa \int^{v} d v^{\prime} \sqrt{F^{\prime 2}\left(v^{\prime}\right)-F^{\prime \prime}\left(v^{\prime}\right)-\frac{1}{2} \gamma\left(v^{\prime}\right)}$,

$\bar{G}(u)= \pm 2 \kappa \int^{u} d u^{\prime} \sqrt{\bar{F}^{\prime 2}\left(u^{\prime}\right)-\bar{F}^{\prime \prime}\left(u^{\prime}\right)-\frac{1}{2} \beta\left(u^{\prime}\right)}$.

Since we are considering the geometry deep inside the neck, where the areal radius is significantly larger than the Schwarzschild radius $a$, Eq. (5.5) is approximated by

$$
\begin{aligned}
R^{2} & =G(v)+\bar{G}(u)-\kappa \log R+\mathcal{O}\left(\kappa^{2}\right), \\
& =G(v)+\bar{G}(u)-\frac{\kappa}{2} \log (G(v)+\bar{G}(u))+\mathcal{O}\left(\kappa^{2}\right),
\end{aligned}
$$

where some constants are absorbed by redefining $G(v)$ and $\bar{G}(u)$. Using Eq. (5.4), $\rho$ is given by 
$\rho=F(v)+\bar{F}(v)-\frac{1}{2 \kappa}(G(v)+\bar{G}(u))-\frac{1}{2} \log R+\mathcal{O}(\kappa)$.

The integration constants $F(v)$ and $\bar{F}(u)$ are related to the choice of the null coordinates $u$ and $v$. We consider the coordinate transformation to $(U, V)$-coordinates for which the metric is expressed as

$$
d s^{2}=\tilde{C} d U d V+R^{2} d \Omega^{2},
$$

where $\tilde{C}$ is related to $C$ via

$$
\tilde{C}=\frac{\partial u}{\partial U} \frac{\partial v}{\partial V} C
$$

Then, $\rho$ transforms to $\tilde{\rho}=\frac{1}{2} \log \tilde{C}$ as

$$
\tilde{\rho}=\rho-\frac{1}{2} \log \left(\frac{d U}{d u}\right)-\frac{1}{2} \log \left(\frac{d V}{d v}\right) .
$$

The solution for $\rho$ is expressed as

$$
\tilde{\rho}=-\frac{R^{2}}{2 \kappa}+\tilde{F}(V)+\overline{\tilde{F}}(U),
$$

where the integration constants $F(v)$ and $\bar{F}(u)$ are transformed to $\tilde{F}(V)$ and $\overline{\tilde{F}}(U)$ as

$$
\begin{aligned}
& F(v)=\tilde{F}(V)+\frac{1}{2} \log \left(\frac{d V}{d v}\right), \\
& \bar{F}(u)=\overline{\tilde{F}}(U)-\frac{1}{2} \log \left(\frac{d U}{d u}\right) .
\end{aligned}
$$

The solution for $R^{2}$ is given by the same equation (5.5) but now $G$ and $\bar{G}$ should be replaced by those for the new coordinates $U$ and $V$ as

$$
\tilde{G}(V)= \pm 2 \kappa \int^{V} d V^{\prime} \sqrt{\tilde{F}^{\prime 2}\left(V^{\prime}\right)-\tilde{F}^{\prime \prime}\left(V^{\prime}\right)-\frac{1}{2} \tilde{\gamma}\left(V^{\prime}\right)},
$$

$$
\overline{\tilde{G}}(U)= \pm 2 \kappa \int^{U} d U^{\prime} \sqrt{\overline{\tilde{F}}^{\prime 2}\left(U^{\prime}\right)-\overline{\tilde{F}}^{\prime \prime}\left(U^{\prime}\right)-\frac{1}{2} \tilde{\beta}\left(U^{\prime}\right)} .
$$

Since the first terms in the expressions (2.4) and (2.5) are not covariant, $\tilde{\beta}$ and $\tilde{\gamma}$ receive corrections under the coordinate transformation as

$$
\beta \rightarrow \tilde{\beta}=\left(\frac{d u}{d U}\right)^{2}\left(\beta+\frac{1}{2}\{U, u\}\right),
$$

$$
\gamma \rightarrow \tilde{\gamma}=\left(\frac{d v}{d V}\right)^{2}\left(\gamma+\frac{1}{2}\{V, v\}\right)
$$

where $\{f, x\}$ is the Schwarzian derivative of $f$ with respect to $x$. By using Eqs. (5.14), (5.15), (5.18), and (5.19), it is straightforward to see that, according to their definitions (5.16), (5.17), (5.6), and (5.7),

$$
G(v)=\tilde{G}(V), \quad \bar{G}(u)=\overline{\tilde{G}}(U) .
$$

\section{B. Junction condition}

The solution (5.4)-(5.7) of the semiclassical Einstein equation for the region deep inside the neck is given in terms of two arbitrary functions $F(v)$ and $\bar{F}(u)$, which are related to the definition of the coordinates $v$ and $u$, respectively. They can be determined by fixing the coordinates. The relation between the coordinate in this deep region and that in the outer region is determined by the junction condition of the metric. In this subsection, we shall choose the same coordinates $u$ and $v$ in the asymptotically flat region, which is defined as $u=t-r$ and $v=t+r$ in the asymptotic Minkowski space. Near the Schwarzschild radius, they are related to the tortoise coordinate $x$ in Sec. II-III via (3.7).

\section{Static case}

We first consider the static case and check that the static solution in Sec. II B is a special case of the general dynamical solution obtained above. The static solution is independent of the time $t$, and the null coordinates can be chosen such that $u=t-x$ and $v=t+x$. Since $F(v)+$ $\bar{F}(u)$ is independent of $t$, we have

$$
F(v)=k_{0}+\frac{1}{4 \kappa} k_{1} v, \quad \bar{F}(u)=\bar{k}_{0}-\frac{1}{4 \kappa} k_{1} u,
$$

where $k_{0}, \bar{k}_{0}$, and $k_{1}$ are constants. Since the null coordinates $u$ and $v$ are chosen such that they are identical to those in the asymptotically flat region, we have $\beta(u)=$ $\gamma(v)=0$ for the static solution. Substituting this static condition (5.21) to Eqs. (5.4)-(5.7) the static solution is obtained as

$$
\begin{gathered}
\rho=k_{0}+\bar{k}_{0}+\kappa^{-1} k_{1} x, \\
R^{2}=k_{0}+\bar{k}_{0}+k_{2}-k_{1} x,
\end{gathered}
$$

where the signs in Eqs. (5.6) and (5.7) are chosen such that $R^{2}$ is independent of $t$, and that $\rho$ is nontrivial. ${ }^{17}$ This is consistent with Eqs. (2.30) and (2.31). After patching the

\footnotetext{
${ }^{17}$ There is another solution for which $\rho$ is a constant at the leading order.
} 
geometry with that near the neck, the constants above are fixed as

$$
k_{0}+\bar{k}_{0} \simeq-\frac{x_{0}}{2 a}, \quad k_{1} \simeq \frac{\kappa}{2 a}, \quad k_{2} \simeq a^{2} .
$$

\section{Dynamical case}

Now, we look for explicit expressions of the metric functions $\rho$ and $R$ for the dynamical case through Eqs. (5.6), (5.7), (5.8), and (5.9) by investigating the junction condition.

In the previous subsection, we have chosen the signs in Eqs. (5.6) and (5.7) by using the static condition of $R^{2}$ and the condition of the nontrivial behavior of $\rho$. However, it is not obvious that $\rho$ should not be a constant, and the signs should be chosen by the junction condition near the neck. For the static case, the radius $R$ inside the neck increases along incoming null lines and decreases along outgoing null lines;

$$
\left(\frac{\partial R^{2}}{\partial v}\right)_{u} \simeq G^{\prime}(v)<0, \quad\left(\frac{\partial R^{2}}{\partial u}\right)_{v} \simeq \bar{G}^{\prime}(u)>0
$$

and the signs in Eqs. (5.6) and (5.7) are consistent with this condition. On the other hand, the geometry for the collapsing shell is constructed by connecting the dynamical solution (5.4)-(5.7) to the flat spacetime through the collapsing shell. The junction condition implies that $R$ on the shell in the geometry (5.4)-(5.7) must be consistent with that in the flat spacetime. As the radius must decrease along the incoming null line in the flat spacetime, $R$ must also decrease in the solution (5.4)-(5.7). Hence, we have the conditions

$$
\left(\frac{\partial R^{2}}{\partial v}\right)_{u} \simeq G^{\prime}(v)<0, \quad\left(\frac{\partial R^{2}}{\partial u}\right)_{v} \simeq \bar{G}^{\prime}(u)<0,
$$

and both signs in Eqs. (5.6) and (5.7) must be minus.

Now, we consider the junction condition to the nearneck region. Near the neck where $R-a=\mathcal{O}(\kappa)$, using Eqs. (3.5), (3.7), we have

$$
\rho=\frac{v-u}{4 a(u)}+\text { const. }+\mathcal{O}(\kappa)
$$

This expression is valid near the neck where $R-a=\mathcal{O}(\kappa)$, and can be extended to the slightly deeper region with $R-a \sim-\kappa \log \kappa$, where $C \sim \mathcal{O}\left(\kappa^{2}\right)$.

Using the expression (5.9), $\rho$ has the following general form deep inside the neck for $R-a \sim \mathcal{O}(a)$ :

$$
\rho=W(v)+\bar{W}(u)-\frac{1}{2} \log R+\mathcal{O}(\kappa) .
$$

This expression is consistent with Eq. (5.27) around the neck. Hence it can be continuously patched with the solution (5.27) over the region from $R-a \sim \mathcal{O}(\kappa)$ to $R-a \sim-\kappa \log \kappa$. The patching requires $W(v)$ and $\bar{W}(u)$ to be approximately given by

$W(v) \simeq \int^{v} \frac{d v^{\prime}}{4 \tilde{a}\left(v^{\prime}\right)}, \quad \bar{W}(u) \simeq-\int^{u} \frac{d u^{\prime}}{4 a\left(u^{\prime}\right)}$,

where $\tilde{a}(v)=a\left(u_{A}(v)\right)$ and $u_{A}(v)$ is the $u$-coordinate of the apparent horizon for a given value of $v .{ }^{18}$ Since the apparent horizon is located at $x \equiv \frac{1}{2}(v-u)=x_{A}, u_{A}(v)$ is given by

$$
u_{A}(v)=v-2 x_{A}(v),
$$

As the time evolution of $x_{A}$ is of $\mathcal{O}(\kappa), u$ - or $v$-dependence of $x_{A}$ can be treated as a higher-order correction. We have used the integration in Eq. (5.29) so that Eq. (5.28) agrees with Eq. (5.27) over a short period of time of $\mathcal{O}(a)$ in which $a(u)$ is almost a constant up to $\mathcal{O}(\kappa)$. After the patching is done, the expression (5.28) holds everywhere inside the neck in vacuum where $C$ is sufficiently small.

We impose the conditions (5.28)-(5.29) to the solution (5.4)-(5.7). For $F(v)$, we impose the initial condition that there is no incoming energy in the past null infinity, $\gamma=0$. For $\bar{F}(u)$, we impose the junction condition on the shell that the outgoing energy is continuous at the shell and hence $\left\langle T_{u и}\right\rangle=0$. Together with these boundary conditions, we obtain

$$
F(v) \simeq \int^{v} \frac{d v^{\prime}}{8 \tilde{a}\left(v^{\prime}\right)}, \quad \bar{F}(u) \simeq-\int^{u} \frac{d u^{\prime}}{4 a\left(u^{\prime}\right)} .
$$

$G(v)$ and $\bar{G}(u)$ are calculated as

$$
G^{\prime}(v) \simeq-2 \kappa F^{\prime}(v) \simeq-\frac{\kappa}{4 \tilde{a}(v)},
$$

$\bar{G}^{\prime}(u) \simeq-2 \kappa \sqrt{-\bar{F}^{\prime \prime}(u)} \simeq-2 \kappa \sqrt{-\frac{\dot{a}(u)}{4 a^{2}(u)}}=\mathcal{O}\left(\kappa^{2}\right)$.

Equation (5.33) implies that the areal radius $R$ is almost constant along the null lines of $v=$ const. By using the expressions above, it is straightforward to see that the formula for the Hawking radiation (3.22) is still valid even if the collapsing shell is deep inside the neck. Thus, we obtain

$$
\bar{F}(u) \simeq \int^{u} 2 \kappa^{-1} a\left(u^{\prime}\right) \dot{a}\left(u^{\prime}\right) d u^{\prime}=\kappa^{-1} a^{2}(u),
$$

and, similarly for $F(v)$ and $G(v)$,

\footnotetext{
${ }^{18}$ It should be noted that $a(u)=a\left(u_{A}(v)\right)$ only at the apparent horizon.
} 


$$
G(v)=2 \kappa F(v)+\text { const. } \simeq \tilde{a}^{2}(v) .
$$

Thus, $\rho$ and $R$ are expressed as

$$
\begin{gathered}
\rho=\kappa^{-1}\left(a^{2}(u)-\tilde{a}^{2}(v)\right)+\frac{\tilde{x}_{A}(u)-x_{0}(u)}{2 a(u)}-\frac{1}{2} \log \tilde{a}(v)+\mathcal{O}(\kappa), \\
R=\tilde{a}(v)+\mathcal{O}(\kappa),
\end{gathered}
$$

where $\tilde{x}_{A}(u)$ is defined by the junction condition such that Eq. (5.36) is consistent with $C$ (3.5) [with Eq. (3.9)] at the apparent horizon, and it is given by

$$
\tilde{x}_{A}(u)=x_{0}(u)-a(u) \log \left(\frac{4 a(u)}{\kappa}\right) .
$$

Hence, $C$ is now obtained as ${ }^{19}$

$$
C \simeq \frac{\kappa}{4 a(u) \tilde{a}(v)} e^{2\left(a^{2}(u)-\tilde{a}^{2}(v)\right) / \kappa} .
$$

The scalar curvature $\mathcal{R}$, squares of the Ricci tensor $\mathcal{R}_{\mu \nu}$ and Riemann tensor $\mathcal{R}_{\mu \nu \rho \sigma}$ are estimated as

$$
\mathcal{R} \simeq \frac{2}{\tilde{a}^{2}(v)}, \quad \mathcal{R}_{\mu \nu} \mathcal{R}^{\mu \nu} \simeq \frac{2}{\tilde{a}^{4}(v)}, \quad \mathcal{R}_{\mu \nu \rho \sigma} \mathcal{R}^{\mu \nu \rho \sigma} \simeq \frac{4}{\tilde{a}^{4}(v)} .
$$

The expressions of the curvature invariants above are valid deep inside the apparent horizon. For a given value of $v$ outside the collapsing shell, as long as $\tilde{a}(v)=a\left(u_{A}(v)\right)$ is large, the curvature is small for any $u>u_{A}(v)$, even when $a(u)$ is approaching the Planck scale.

\section{Collapsing shell deep inside the neck}

In the previous section, we have seen that the areal radius is almost constant along the incoming null lines, $v=$ const. Although $R$ is decreasing along the collapsing shell, its decreasing rate is of $\mathcal{O}\left(\kappa^{2}\right)$ and the Schwarzschild radius $a(u)$ is decreasing much faster. This situation continues as long as $a(u)$ is sufficiently larger than the Planck length. Hence, when $a(u)$ becomes much smaller than its initial value (but still larger than the Planck length), the areal radius of the shell is still large at the same time $u$, so the interior space inside the neck (including the space occupied by the collapsing matter) is still large. Naively, this large interior space inside the neck either becomes disconnected from the outside world as the neck shrinks to 0 eventually, resulting in the event horizon, or they remain connected to the outside world through tiny necks as remnants.

\footnotetext{
${ }^{19}$ Note that $a^{2}(u)-a^{2}\left(u_{A}(v)\right)<0$ for $(u, v)$ inside the apparent horizon.
}

However, the expression (5.33) implies that the decreasing rate of the areal radius along the incoming null lines can be large when the Schwarzschild radius $a$ approaches the Planck length $\ell_{p}$. The semiclassical approximation will be invalid, and the outgoing energy of the Hawking radiation $\left(\sim 1 / a^{2}\right)$ will be at the Planck scale. Although the expression (5.33) would not be reliable when $a \sim \ell_{p}$, there would not be the problem of the cutoff scale if we focus on the locus of the collapsing shell. When the areal radius starts decreasing again, it is still much larger than the Planck length and comparable to the Schwarzschild radius for the initial mass of the shell. The outgoing energy $T_{u u}$ is also zero just on the shell. Thus it would be possible to study the behavior of the shell without referring to $a(u)$.

In this subsection, we check that the areal radius at the shell starts to decrease again. Note that the new coordinate for $u$-direction at the shell cannot be connected to that in the asymptotic region when $a(u)=\mathcal{O}(\kappa)$, since the semiclassical approximation would not be valid around the apparent horizon in between.

By choosing a suitable coordinate system $(\tilde{u}, \tilde{v})$, we can set $\tilde{F}(\tilde{v})=0$ and $\overline{\tilde{F}}(\tilde{u})=0$, so that the solution is simply given by [for $R^{2} \gg \mathcal{O}(\alpha)$ ]

$$
\begin{gathered}
\rho \simeq-\frac{R^{2}}{2 \kappa}, \\
R^{2} \simeq G(\tilde{v})+\bar{G}(\tilde{u}),
\end{gathered}
$$

where

$$
\begin{aligned}
& G(\tilde{v})=-2 \kappa \int^{\tilde{v}} d v^{\prime} \sqrt{-\frac{1}{2} \tilde{\gamma}\left(v^{\prime}\right)} \\
& \bar{G}(\tilde{u})=-2 \kappa \int^{\tilde{u}} d u^{\prime} \sqrt{-\frac{1}{2} \tilde{\beta}\left(u^{\prime}\right),}
\end{aligned}
$$

Note that the coordinates $\tilde{u}$ and $\tilde{v}$ in the expressions above are different from the original null coordinates, and $\tilde{\gamma}(\tilde{v})$ and $\tilde{\beta}(\tilde{u})$ are different from the incoming and outgoing radiation in the asymptotically flat past and future null infinities [see Eqs. (5.18), (5.19)]. The outgoing energymomentum tensor is calculated as

$$
R^{2}\left\langle T_{\text {ии }}\right\rangle=2 \tilde{\beta}(\tilde{u})+(-2 \tilde{\beta}(\tilde{u}))^{-1 / 2} \dot{\tilde{\beta}}(\tilde{u}) .
$$

Since $\left\langle T_{\text {ии }}\right\rangle$ must be continuous across the incoming null shell, we impose the junction condition $\left\langle T_{u и}\right\rangle=0$, and then, $\tilde{\beta}(\tilde{u})$ is solved as

$$
\tilde{\beta}=-\frac{1}{2\left(\tilde{u}-\tilde{u}_{0}\right)^{2}} .
$$

The solution for $\beta$ is negative, so that $\bar{G}(\tilde{u})$ is real. $\bar{G}^{\prime}(\tilde{u})$ is monotonically increasing or decreasing depending on 
whether $\tilde{u}>\tilde{u}_{0}$ or $\tilde{u}<\tilde{u}_{0}$. By comparing with (5.33), $\bar{G}^{\prime}(\tilde{u})$ should decrease with time. In this case, the areal radius $R$ on the shell behaves as

$$
R^{2}=\bar{G}(\tilde{u})+\text { const. } \simeq \kappa \log \left(\tilde{u}_{0}-\tilde{u}\right)+\text { const. },
$$

which goes to zero at some point, $\tilde{u}=\tilde{u}_{e}<\tilde{u}_{0}$. Therefore, the collapsing shell eventually reaches $R=0$ in the exterior geometry.

\section{Distance between apparent horizon and collapsing shell}

In this subsection, using the explicit metric derived above deep inside the horizon, we will show that the proper distance from the shell to the apparent horizon is indeed very short even when the collapsing shell has moved to the deeper region.

Now, we calculate the proper distance between the collapsing shell and the apparent horizon. Using the explicit form of (5.39), we can see that the $(u, v)$-subspace is $2 \mathrm{D}$ flat spacetime:

$d s^{2}=\frac{\kappa}{4 a(u) \tilde{a}(v)} e^{2\left(a^{2}(u)-\tilde{a}^{2}(v)\right) / \kappa} d u d v=\kappa d U d V$,

where the new coordinates $U$ and $V$ are defined as

$$
\begin{aligned}
& U(u)=-\int_{u}^{u_{c}} \frac{1}{2 a\left(u^{\prime}\right)} e^{2 a^{2}\left(u^{\prime}\right) / \kappa} d u^{\prime}, \\
& V(v)=\int_{v_{s}}^{v} \frac{1}{2 \tilde{a}(v)} e^{-2 \tilde{a}^{2}\left(v^{\prime}\right) / \kappa} d v^{\prime},
\end{aligned}
$$

where $v=v_{s}$ is the position of the collapsing null shell, and $u=u_{c}$ is the retarded time at the evaporation. ${ }^{20}$ Recall that $a(u)$ and $\tilde{a}(v)=a\left(u_{A}(v)\right)$ stand for the areal radius of the apparent horizon at $u$ and $v$, respectively, and hence, satisfy $a^{2}(u)-\tilde{a}^{2}(v) \leq 0$ since $a$ is decreasing with time. By using the formula of the Hawking radiation (3.22), $U$ and $V$ are expressed as

$$
U(u)=\left(1-e^{2 a^{2}(u) / \kappa}\right), \quad V(v)=\left(e^{-2 \tilde{a}^{2}(v) / \kappa}-e^{-2 a_{0}^{2} / \kappa}\right),
$$

where $a_{0}$ is the Schwarzschild radius for the initial mass of the shell, or equivalently, the maximum of $a$.

Consider two arbitrary points $(u, v)$ and $\left(u^{\prime}, v^{\prime}\right)$ inside the apparent horizon with a spacelike separation. Their proper distance $L$ is bounded from above by

\footnotetext{
${ }^{20}$ To be more precise, $u_{c}$ should be the cutoff time when the Schwarzschild radius $a(u)$ becomes sufficiently small but still larger than the Planck scale.
}

$$
\begin{aligned}
L^{2}= & -\kappa\left(U(u)-U\left(u^{\prime}\right)\right)\left(V(v)-V\left(v^{\prime}\right)\right) \\
& <\kappa\left(e^{2 a^{2}(u) / \kappa}-e^{2 a^{2}\left(u^{\prime}\right) / \kappa}\right)\left(e^{-2 \tilde{a}^{2}(v) / \kappa}-e^{-2 \tilde{a}^{2}\left(v^{\prime}\right) / \kappa}\right) \\
& <\kappa e^{2\left(a^{2}(u)-\tilde{a}^{2}(v)\right) / \kappa} \leq \kappa=\ell_{p}^{2} .
\end{aligned}
$$

(Without loss of generality, we assume that $\left(u^{\prime}, v^{\prime}\right)$ is deeper inside the horizon than $(u, v)$, i.e., $u^{\prime}>u$ and $v^{\prime}<v$.) Therefore, the proper distance between two arbitrary points inside the apparent horizon is of $\mathcal{O}\left(\ell_{p}\right)$.

\section{CONCLUSION AND DISCUSSION}

In this paper, we have studied effects of the vacuum energy-momentum tensor in the formation and evaporation of a black hole. We have considered a thin shell which collapses at the speed of light as a convenient idealization. The geometry is obtained by connecting the flat spacetime inside the shell with the black-hole geometry outside the shell.

We have focused on the spherically symmetric configurations, and used the s-wave approximation. The vacuum energy-momentum tensor is assumed to be approximated by that of the 2-dimensional scalar field, so all angular components vanish. ${ }^{21}$ The geometry is then uniquely fixed by the initial condition in the past null infinity and the junction condition at the collapsing shell. By taking the initial condition such that the incoming energy is absent in the asymptotic region, the negative vacuum energy appears near the Schwarzschild radius, and the areal radius has a local minimum slightly larger than the Schwarzschild radius on each null surface of constant retarded time $(u=$ const $)$.

Assuming that the angular components of the energymomentum tensor vanish on the collapsing null shell, the outgoing energy $T_{и и}$ must be continuous across the shell, and hence it has to be zero just outside the shell. This implies that the Hawking radiation can take no energy directly from the shell. Furthermore, the quantum energymomentum tensor on top of the shell is positive and increasing such that the total (delta functional) incoming energy on the shell is increasing. The shell collapses without losing its mass.

The presence of the negative energy outside the shell leads to the structure of Wheeler's bag of gold. The neck of the bag is nothing but the local minimum of the areal radius. It also plays the role of the apparent horizon in the time-dependent case. If the neck continues to shrink and finally closes at some point, the interior of the neck is disconnected from the external universe, and there would be an event horizon. However, the semiclassical Einstein equation is only the low-energy effective theory for scales much larger than the Planck length. The neck

\footnotetext{
${ }^{21}$ This model for the vacuum energy-momentum tensor is widely studied in the literature for 4D black-hole geometry; see, e.g., $[1,5,7,15-17]$.
} 

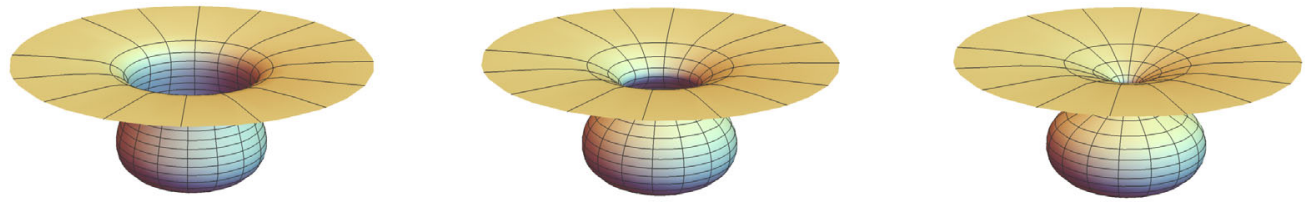

FIG. 4. The schematic diagrams for an evaporating black hole that ends up with a Planckian neck and a large internal space [12].
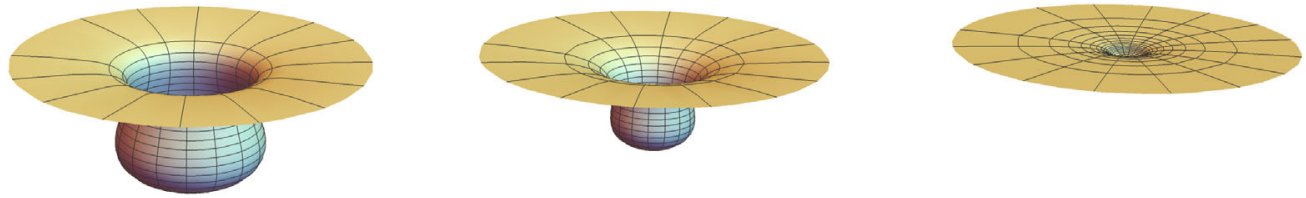

FIG. 5. The schematic diagrams for an evaporating black hole for which the interior space shrinks together with the neck [12].

may stop shrinking at a finite size possibly of the Planck scale. We cannot tell within the framework of the lowenergy effective theory whether or not there would be an event horizon.

It is interesting to know whether the scale of the interior space is still large when the black hole is nearly completely evaporated. Our results show that the decreasing rate of the areal radius of the collapsing shell becomes nearly zero while the difference of the areal radii between the shell and the horizon becomes large. That is, the areal radius of the shell can become much larger than that at the apparent horizon at the same time (in terms of the $u$-coordinate). In [12], it was argued that there are two possible scenarios depending on whether the size of the interior space shrinks together with the neck, which are shown in Figs. 4 and 5. Our result in this paper implies that in terms of the areal radius, the scenario of Fig. 4 is realized for the gravitational collapse of the thin shell. This is related to the fact that the energy of the shell cannot be turned into Hawking radiation in the absence of high-energy events, and the shell keeps its initial mass until it can no longer be described in the lowenergy effective theory. A crucial assumption related to this conclusion is that the energy-momentum tensor on the shell does not have nonzero angular components. If the collapsing shell has tangential pressure in the angular directions, the outgoing energy flux can be discontinuous across the shell without violating energy conservation. In this case, it is possible that the outgoing energy appears just outside the shell and the Hawking radiation can directly take the energy of the shell away, as the case of the model proposed in Refs. [18,19].

As the proper size inside the collapsing shell is determined by its areal radius since the spacetime is flat inside the shell, the size of the space inside the apparent horizon is much larger than what is suggested by the size of the neck when a large portion of the black-hole mass is evaporated.

Our calculation suggests that the proper distance between the collapsing shell and the apparent horizon is as small as the Planck length. ${ }^{22}$ The positive energy of the shell and the negative vacuum energy are placed within this small region of the Planck scale. In particular, most of the vacuum negative energy is placed in the deeper region whose size in proper length is much smaller than the Planck scale. Since the semiclassical picture of the spacetime is not good for such a small region, the positive and negative energy in this small region should be course-grained as a more appropriate picture of the spacetime in the low-energy effective theory.

Strictly speaking, the Planck-scale separation between the apparent horizon and the shell means that we cannot really distinguish the location of the collapsing shell from that of the neck in the context of a low-energy effective theory with a cutoff length scale larger than the Planck length. Whether the collapsing matter has entered the apparent horizon is thus a question beyond the scope of low-energy effective theories. We can only conclude that the apparent horizon and the collapsing shell are approximately around the same place. It is hence interesting to note that, in the model proposed in Refs. [18,19] (with a different assumption about the vacuum energy-momentum tensor), the collapsing matter stays outside the apparent horizon by a Planckian distance, so that in fact the apparent horizon does not emerge. The difference between models with and without apparent horizons might be subtler than what people have expected.

\section{ACKNOWLEDGMENTS}

The author would like to thank Hikaru Kawai, Piljin Yi, Tatsuma Nishioka for discussions. P. M. H. thanks iTHEMS at RIKEN, University of Tokyo and Kyoto University for their hospitality during his visits where a significant part of this work was done. P. M. H. is supported

\footnotetext{
${ }^{22} \mathrm{~A}$ concise proof of the Planckian proper distance between the collapsing shell and the trapping horizon for a wider class of models will be given elsewhere [20].
} 
in part by the Ministry of Science and Technology, R. O.C. and by National Taiwan University. The work of Y. M. is supported in part by JSPS KAKENHI Grants No. JP17H06462. Y. Y. is partially supported by Japan
Society of Promotion of Science (JSPS), Grants-in-Aid for Scientific Research (KAKENHI) Grants No. 18K13550 and 17 H01148. Y. Y. is also partially supported by RIKEN iTHEMS Program.
[1] P. C. W. Davies, S. A. Fulling, and W. G. Unruh, Energymomentum tensor near an evaporating black hole, Phys. Rev. D 13, 2720 (1976).

[2] S. M. Christensen and S. A. Fulling, Trace anomalies and the Hawking effect, Phys. Rev. D 15, 2088 (1977).

[3] S. D. Mathur, The information paradox: A pedagogical introduction, Classical Quantum Gravity 26, 224001 (2009).

[4] A. Almheiri, D. Marolf, J. Polchinski, and J. Sully, Black holes: Complementarity or firewalls?, J. High Energy Phys. 02 (2013) 062; S. L. Braunstein, Black hole entropy as entropy of entanglement, or it's curtains for the equivalence principle, arXiv:0907.1190v1; S. L. Braunstein, S. Pirandola, and K. Życzkowski, Better Late than Never: Information Retrieval from Black Holes, Phys. Rev. Lett. 110, 101301 (2013) for a similar prediction from different assumptions.

[5] R. Parentani and T. Piran, The Internal Geometry of an Evaporating Black Hole, Phys. Rev. Lett. 73, 2805 (1994).

[6] S. N. Solodukhin, Can black hole relax unitarily?, arXiv: hep-th/0406130; S. N. Solodukhin, Restoring unitarity in BTZ black hole, Phys. Rev. D 71, 064006 (2005); T. Damour and S. N. Solodukhin, Wormholes as black hole foils, Phys. Rev. D 76, 024016 (2007).

[7] A. Fabbri, S. Farese, J. Navarro-Salas, G. J. Olmo, and H. Sanchis-Alepuz, Semiclassical zero-temperature corrections to Schwarzschild spacetime and holography, Phys. Rev. D 73, 104023 (2006); Static quantum corrections to the Schwarzschild spacetime, J. Phys. Conf. Ser. 33, 457 (2006).

[8] P. M. Ho and Y. Matsuo, Static black holes with back reaction from vacuum energy, Classical Quantum Gravity 35, 065012 (2018).

[9] P. M. Ho and Y. Matsuo, Static black hole and vacuum energy: Thin shell and incompressible fluid, J. High Energy Phys. 03 (2018) 096.

[10] C. Berthiere, D. Sarkar, and S. N. Solodukhin, The fate of black hole horizons in semiclassical gravity, Phys. Lett. B 786, 21 (2018).
[11] P. M. Ho, H. Kawai, Y. Matsuo, and Y. Yokokura, Back reaction of 4D conformal fields on static geometry, J. High Energy Phys. 11 (2018) 056.

[12] P. M. Ho and Y. Matsuo, On the near-horizon geometry of an evaporating black hole, J. High Energy Phys. 07 (2018) 047.

[13] S. A. Fulling and P. C. W. Davies, Radiation from a moving mirror in two-dimensional space-time: conformal anomaly, Proc. R. Soc. A 348, 393 (1976).

[14] P. M. Ho, Y. Matsuo, and S. J. Yang, Asymptotic states of black holes in KMY model, Classical Quantum Gravity 37, 035002 (2020).

[15] R. Brout, S. Massar, R. Parentani, and P. Spindel, A primer for black hole quantum physics, Phys. Rep. 260, 329 (1995).

[16] S. Ayal and T. Piran, Spherical collapse of a massless scalar field with semiclassical corrections, Phys. Rev. D 56, 4768 (1997).

[17] C. Barcelo, S. Liberati, S. Sonego, and M. Visser, Fate of gravitational collapse in semiclassical gravity, Phys. Rev. D 77, 044032 (2008).

[18] H. Kawai, Y. Matsuo, and Y. Yokokura, A self-consistent model of the black hole evaporation, Int. J. Mod. Phys. A 28, 1350050 (2013).

[19] H. Kawai and Y. Yokokura, Phenomenological description of the interior of the Schwarzschild black hole, Int. J. Mod. Phys. A 30, 1550091 (2015); P. M. Ho, Comment on selfconsistent model of black hole formation and evaporation, J. High Energy Phys. 08 (2015) 096; H. Kawai and Y. Yokokura, Interior of black holes and information recovery, Phys. Rev. D 93, 044011 (2016); P. M. Ho, The absence of horizon in black-hole formation, Nucl. Phys. B909, 394 (2016); Asymptotic black holes, Classical Quantum Gravity 34, 085006 (2017); H. Kawai and Y. Yokokura, A model of black hole evaporation and 4D Weyl anomaly, Universe 3, 51 (2017).

[20] P. M. Ho, Y. Matsuo, and Y. Yokokura, Distance between collapsing matter and trapping horizon in evaporating black holes, arXiv:1912.12863. 